ESAIM: COCV 26 (2020) 55

https://doi.org/10.1051/cocv/2019033
ESAIM: Control, Optimisation and Calculus of Variations

www.esaim-cocv.org

\title{
LOCAL CONTROLLABILITY OF REACTION-DIFFUSION SYSTEMS AROUND NONNEGATIVE STATIONARY STATES
}

\author{
KÉVIN LE BALC'H*
}

\begin{abstract}
We consider a $n \times n$ nonlinear reaction-diffusion system posed on a smooth bounded domain $\Omega$ of $\mathbb{R}^{N}$. This system models reversible chemical reactions. We act on the system through $m$ controls $(1 \leq m<n)$, localized in some arbitrary nonempty open subset $\omega$ of the domain $\Omega$. We prove the local exact controllability to nonnegative (constant) stationary states in any time $T>0$. A specificity of this control system is the existence of some invariant quantities in the nonlinear dynamics that prevents controllability from happening in the whole space $L^{\infty}(\Omega)^{n}$. The proof relies on several ingredients. First, an adequate affine change of variables transforms the system into a cascade system with second order coupling terms. Secondly, we establish a new null-controllability result for the linearized system thanks to a spectral inequality for finite sums of eigenfunctions of the Neumann Laplacian operator, due to David Jerison, Gilles Lebeau and Luc Robbiano and precise observability inequalities for a family of finite dimensional systems. Thirdly, the source term method, introduced by Yuning Liu, Takéo Takahashi and Marius Tucsnak, is revisited in a $L^{\infty}$-context. Finally, an appropriate inverse mapping theorem in suitable spaces enables to go back to the nonlinear reaction-diffusion system.
\end{abstract}

Mathematics Subject Classification. 93B05 35K51 35K57 35K58 93C20.

Received September 28, 2018. Accepted April 26, 2019.

\section{INTRODUCTION}

\subsection{Free system}

Let $n \geq 2$ be an integer. We consider the following reversible chemical reaction:

$$
\alpha_{1} A_{1}+\cdots+\alpha_{n} A_{n} \rightleftharpoons \beta_{1} A_{1}+\cdots+\beta_{n} A_{n},
$$

where $A_{1}, \ldots, A_{n}$ denote $n$ chemical species and $\left(\alpha_{1}, \ldots, \alpha_{n}\right),\left(\beta_{1}, \ldots, \beta_{n}\right) \in(\mathbb{N})^{n}$ are such that for every $1 \leq i \leq n, \alpha_{i} \neq \beta_{i}$. Chemically, according to the forward reaction $\rightarrow$ of (1.1), when $\alpha_{i}$ molecules of $A_{i}$ disappear $(1 \leq i \leq n)$, they are called the "reactants", then $\beta_{i}$ molecules of $A_{i}$ appear $(1 \leq i \leq n)$. The backward reaction $\angle$ of (1.1) is governed by the same law: when $\beta_{i}$ molecules of $A_{i}$ disappear $(1 \leq i \leq n)$, here they are the reactants, then $\alpha_{i}$ molecules of $A_{i}$ appear $(1 \leq i \leq n)$.

For $1 \leq i \leq n$, let $u_{i}(t,):. \Omega \rightarrow \mathbb{R}$ be the concentration of the chemical component $A_{i}$ at time $t$. The law of mass action states that the rate of a chemical reaction is directly proportional to the product of the

Keywords and phrases: Controllability, reaction-diffusion system, nonlinear coupling.

ENS Rennes, Université de Rennes, CNRS, IRMAR - UMR 6625, 35000 Rennes, France.

* Corresponding author: kevin.lebalch@ens-rennes.fr 
concentrations of the reactants. Using this law together with the Fick's law for the diffusion of the components, we obtain that $u_{i}$ satisfies the following reaction rate equation (see e.g. [30], Sect. 1.2):

$$
\begin{gathered}
\partial_{t} u_{i}-\underbrace{d_{i} \Delta u_{i}}_{\text {diffusion }}+\underbrace{\beta_{i} \prod_{k=1}^{n} u_{k}^{\alpha_{k}}}_{\begin{array}{c}
\text { loss of forward reacting molecules } \\
\alpha_{i} \prod_{k=1}^{n} u_{k}^{\alpha_{k}}
\end{array}}+\underbrace{\beta_{i} \prod_{k=1}^{n} u_{k}^{\beta_{k}}}_{\text {gain of of backward reacting molecules }} \\
=\underbrace{\alpha_{i} \prod_{k=1}^{n} u_{k}^{\beta_{k}}}_{\text {gain of backward reacting molecules }}
\end{gathered}
$$

that is to say,

$$
\partial_{t} u_{i}-d_{i} \Delta u_{i}=\left(\beta_{i}-\alpha_{i}\right)\left(\prod_{k=1}^{n} u_{k}^{\alpha_{k}}-\prod_{k=1}^{n} u_{k}^{\beta_{k}}\right),
$$

where $d_{i} \in(0,+\infty)$ is the diffusion coefficient of the chemical species $A_{i}$.

For a given matrix $M$, we introduce the notation $M^{\text {tr }}$ for the transpose of the matrix $M$.

From (1.2), by setting

$$
U:=\left(u_{1}, \ldots, u_{n}\right)^{\mathrm{tr}}
$$

we deduce that $U$ satisfies the following reaction-diffusion system:

$$
\begin{cases}\partial_{t} U-D \Delta U=F(U) & \text { in }(0, T) \times \Omega \\ \frac{\partial U}{\partial \nu}=0 & \text { on }(0, T) \times \partial \Omega \\ U(0, .)=u_{0} & \text { in } \Omega\end{cases}
$$

where

$$
\begin{gathered}
D:=\operatorname{diag}\left(d_{1}, \ldots, d_{n}\right), \\
F(U):=\left(f_{i}\left(u_{1}, \ldots, u_{n}\right)\right)_{1 \leq i \leq n}^{\operatorname{tr}},
\end{gathered}
$$

with

$$
\forall 1 \leq i \leq n, f_{i}\left(u_{1}, \ldots, u_{n}\right):=\left(\beta_{i}-\alpha_{i}\right)\left(\prod_{k=1}^{n} u_{k}^{\alpha_{k}}-\prod_{k=1}^{n} u_{k}^{\beta_{k}}\right)
$$

and $T \in(0,+\infty), \Omega$ is a bounded, connected, open subset of $\mathbb{R}^{N}$ (with $N \geq 1$ ) of class $C^{2}, \nu$ is the outer unit normal vector to $\partial \Omega$.

In general, global existence of classical solutions (in the sense of [31], Def. 1.5) or weak solutions (in the sense of [31], Def. 5.12 replacing $\geq$ by $=$ ) for (1.3) with $F$, defined as in (1.5), (1.6), is an open problem.

- For particular semilinearities with a so-called triangular structure (see [31], Sect. 3.3), classical solutions exist in the time interval $\left(0,+\infty\right.$ and are unique. For example, take $n=2, \alpha_{1} \geq 1, \beta_{2}=1, \alpha_{2}=\beta_{1}=0$ and apply [31], Thm. 3.1). 
- For at most quadratic nonlinearities, global existence of weak solutions holds (see [31], Thm. 5.12). For instance, take $n=4, \alpha_{1}=\alpha_{3}=\beta_{2}=\beta_{4}=1, \alpha_{2}=\alpha_{4}=\beta_{1}=\beta_{3}=0$. For any spatial dimension $N \geq 1$, the recent works [6] and [33] (inspired by the previous works [21] and [22]) prove that the solutions are bounded for bounded initial data, which ensure global existence of classical solutions.

- Without a priori $L^{1}$-bound on the nonlinearities, a challenging problem is to understand whether global solutions exist. For example, take $n=2, \alpha_{1}=\beta_{2}=2, \beta_{1}=\alpha_{2}=3$ (see [31], Prob. 1).

Let us also mention that global existence of renormalized solutions holds in all cases for (1.3) (see [15]).

\subsection{Control system and open question}

We assume that one can act on the system through controls localized on a nonempty open subset $\omega$ of $\Omega$. From a chemical viewpoint, it means that one can add or remove chemical species at a specific location of the domain $\Omega$. More precisely, let

$$
J \subset\{1, \ldots, n\} \text { and } m:=\# J \leq n \text { be the number of controls. }
$$

Up to a renumbering $\left(u_{i}\right)_{1 \leq i \leq n}$, we can assume that $J=\{1, \ldots, m\}$ where $J$ is defined in (1.7). Hence, we define

$$
H^{J}:=\left(h_{1}, \ldots, h_{m}, 0, \ldots, 0\right)^{\mathrm{tr}} .
$$

We consider the control system:

$$
\begin{cases}\partial_{t} U-D \Delta U=F(U)+H^{J} 1_{\omega} & \text { in }(0, T) \times \Omega \\ \frac{\partial U}{\partial \nu}=0 & \text { on }(0, T) \times \partial \Omega \\ U(0, .)=U_{0} & \text { in } \Omega .\end{cases}
$$

Here, at $t \in[0, T], U(t,):. \Omega \rightarrow \mathbb{R}^{n}$ is the state to be controlled, $H^{J}(t,):. \Omega \rightarrow \mathbb{R}^{m}$ is the control input supported in $\omega$.

Let

$$
U^{*}:=\left(u_{1}^{*}, \ldots, u_{n}^{*}\right)^{\operatorname{tr}}
$$

be a nonnegative stationary state of (1.3) i.e.

$$
\forall 1 \leq i \leq n, u_{i}^{*} \in[0,+\infty) \text { and } \prod_{k=1}^{n} u_{k}^{* \alpha_{k}}=\prod_{k=1}^{n} u_{k}^{* \beta_{k}} .
$$

Note that the nonnegative stationary solutions of (1.3) do not depend on the space variable (see Prop. A.6 in Appendix A.2). Thus, it is not restrictive to assume that $U^{*} \in[0,+\infty)^{n}$.

The question we ask is the following one: For a given initial condition $U_{0}$, does there exist $H^{J}$ such that the solution $U$ of (NL-U) satisfies

$$
\forall i \in\{1, \ldots, n\}, u_{i}(T, .)=u_{i}^{*} ?
$$

Under appropriate assumptions (see Assumptions 1.4 and 1.6), we prove the controllability of (NL-U), in an appropriate subspace of $L^{\infty}(\Omega)^{n}$, locally around $U^{*}$, with controls in $L^{\infty}((0, T) \times \Omega)^{m}$ (see Thm. 1.7).

By an adequate affine transformation, the proof relies on the study of the null-controllability of an equivalent cascade system with second order coupling terms (see Sect. 2.1). 
We have chosen to postpone the simple or classical proofs in Appendix A. Therefore, the main contributions are highlighted in the body of the article.

\subsection{Nonlinear well-posedness result}

For $\tau>0$, we introduce

$$
Q_{\tau}:=(0, \tau) \times \Omega
$$

We define the function space

$$
W_{T}:=L^{2}\left(0, T ; H^{1}(\Omega)\right) \cap H^{1}\left(0, T ;\left(H^{1}(\Omega)\right)^{\prime}\right),
$$

that satisfies the continuous embedding

$$
W_{T} \hookrightarrow C\left([0, T] ; L^{2}(\Omega)\right)
$$

We introduce the notion of solution associated to the nonlinear system (NL-U) (see Sect. 1.2).

Definition 1.1. Let $D$ be defined in (1.4). For every $U_{0} \in L^{\infty}(\Omega)^{n}, H^{J} \in L^{\infty}\left(Q_{T}\right)^{m}$, we say that $U \in\left(W_{T} \cap\right.$ $\left.L^{\infty}\left(Q_{T}\right)\right)^{n}$ is a solution of (NL-U) if for every $V \in L^{2}\left(0, T ; H^{1}(\Omega)^{n}\right)$,

$$
\int_{0}^{T}\left(\partial_{t} U, V\right)_{\left.\left(H^{1}(\Omega)^{n}\right)^{\prime}, H^{1}(\Omega)^{n}\right)}+\int_{Q_{T}} D \nabla U . \nabla V=\int_{Q_{T}}\left(F(U)+H^{J} 1_{\omega}\right) \cdot V,
$$

with $F$ defined in (1.5) and

$$
U(0, .)=U_{0} \text { in } L^{\infty}(\Omega)^{n}
$$

Remark 1.2. Given $U_{0} \in L^{\infty}(\Omega)^{n}, H^{J} \in L^{\infty}\left(Q_{T}\right)^{m}$, if a solution $U$ of (NL-U) exists in the sense of Definition 1.1, then it is unique because $F$ is locally Lipschitz on $\mathbb{R}^{n}$ (see the proof of [23] Def.-Prop. 2.4).

\subsection{Invariant quantities of the nonlinear dynamics}

In this section, we show that in the system (NL-U) (see Sect. 1.2), when the number of controls is small, some quantities are invariant. They impose some restrictions on the initial condition, for the controllability results.

Proposition 1.3. We assume that $m \leq n-2$. Let $U_{0} \in L^{\infty}(\Omega)^{n}, H^{J} \in L^{\infty}\left(Q_{T}\right)^{m}$. Assume that $U$ is a solution of (NL-U) such that $U(T,)=.U^{*}$ with $U^{*}$ defined in (1.9). Then, we have for every $k \neq l \in\{m+1, \ldots, n\}$, $t \in[0, T]$,

$$
\begin{gathered}
\int_{\Omega} \frac{u_{k}(t, x)-u_{k}^{*}}{\beta_{k}-\alpha_{k}} \mathrm{~d} x=\int_{\Omega} \frac{u_{l}(t, x)-u_{l}^{*}}{\beta_{l}-\alpha_{l}} \mathrm{~d} x, \\
\left(d_{k}=d_{l}\right) \Rightarrow\left(\frac{u_{k}(t, .)-u_{k}^{*}}{\beta_{k}-\alpha_{k}}=\frac{u_{l}(t, .)-u_{l}^{*}}{\beta_{l}-\alpha_{l}}\right) .
\end{gathered}
$$


In particular, for every $k \neq l \in\{m+1, \ldots, n\}$,

$$
\begin{gathered}
\int_{\Omega} \frac{u_{k, 0}(x)-u_{k}^{*}}{\beta_{k}-\alpha_{k}} \mathrm{~d} x=\int_{\Omega} \frac{u_{l, 0}(x)-u_{l}^{*}}{\beta_{l}-\alpha_{l}} \mathrm{~d} x, \\
\left(d_{k}=d_{l}\right) \Rightarrow\left(\frac{u_{k, 0}-u_{k}^{*}}{\beta_{k}-\alpha_{k}}=\frac{u_{l, 0}-u_{l}^{*}}{\beta_{l}-\alpha_{l}}\right) .
\end{gathered}
$$

The proof of Proposition 1.3 is done in Appendix A.3. We prove (1.14) by integrating with respect to the space variable an appropriate linear combination of equations of (NL-U) and by using the Neumann boundary conditions. We prove (1.15) by the backward uniqueness of the heat equation applied to an appropriate linear combination of equations of (NL-U).

An equation (1.15) implies that we can reduce the number of components of $\left(u_{i}\right)_{1 \leq i \leq n}$ of (NL-U) when some diffusion coefficients $d_{i}$ are equal for $m+1 \leq i \leq n$. Thus, (NL-U) becomes more simple under this last assumption. That is why, we make the following hypothesis in order to treat the most difficult case.

Assumption 1.4. For $m \leq n-2$, we suppose that for every $k \neq l \in\{m+1, \ldots, n\}, d_{k} \neq d_{l}$.

Remark 1.5. It will be interesting to note that the mass condition (1.16) is obviously equivalent to

$$
\forall k \geq m+2, \int_{\Omega} \frac{u_{k, 0}(x)-u_{k}^{*}}{\beta_{k}-\alpha_{k}} \mathrm{~d} x=\int_{\Omega} \frac{u_{m+1,0}(x)-u_{m+1}^{*}}{\beta_{m+1}-\alpha_{m+1}} \mathrm{~d} x
$$

\subsection{Main result}

We will work under the following assumption that will ensure the controllability of the linearized system of (NL-U) (see Sect. 2.2).

Assumption 1.6. For $m \leq n-1$, we assume that

$$
\partial_{m} f_{m+1}\left(u_{1}^{*}, \ldots, u_{n}^{*}\right) \neq 0
$$

where $f_{m+1}$ is defined in (1.6).

Theorem 1.7. Under Assumptions 1.4 and 1.6, the system (NL-U) is locally controllable around $U^{*}$, i.e., there exists $r>0$ such that for every $U_{0} \in L^{\infty}(\Omega)^{n}$ satisfying the mass condition (1.16) and $\left\|U_{0}-U^{*}\right\|_{L^{\infty}(\Omega)} \leq r$, there exists $H^{J} \in L^{\infty}\left(Q_{T}\right)^{m}$ such that the solution $U$ of (NL-U) satisfies $U(T,)=.U^{*}$.

Remark 1.8. The uniqueness of the solution $U \in L^{\infty}\left(Q_{T}\right)^{n}$ is a consequence of Remark 1.2. The existence of the solution $U \in L^{\infty}\left(Q_{T}\right)^{n}$ is a consequence of a good choice of the control $H^{J} \in L^{\infty}\left(Q_{T}\right)^{m}$ and more precisely of an inverse mapping argument (see Sect. 6).

Remark 1.9. Up to renumbering the first $m$ equations of (NL-U), we can see that Theorem 1.7 is still valid by replacing the assumption (1.19) by

$$
\exists j \in\{1, \ldots, m\}, \partial_{j} f_{m+1}\left(u_{1}^{*}, \ldots, u_{n}^{*}\right) \neq 0 .
$$

Remark 1.10. When $\alpha_{m}, \beta_{m} \geq 1$, a sufficient condition to ensure (1.19) is

$$
\forall 1 \leq k \leq n, u_{k}^{*} \neq 0
$$


Indeed, by using (1.6), (1.10) and $\alpha_{j} \neq \beta_{j}$, if (1.21) holds true then

$$
\partial_{m} f_{m+1}\left(u_{1}^{*}, \ldots, u_{n}^{*}\right)=\frac{\alpha_{m}-\beta_{m}}{u_{m}^{*}} \prod_{k=1}^{n} u_{k}^{* \alpha_{k}} \neq 0 .
$$

Note that (1.21) is not equivalent to (1.19) as shown by the examples in Application 1.11 (see below).

Application 1.11. For $n=4, \alpha_{1}=\alpha_{3}=\beta_{2}=\beta_{4}=1$ and $\alpha_{2}=\alpha_{4}=\beta_{1}=\beta_{3}=0$, we have

$$
f_{i}\left(u_{1}, u_{2}, u_{3}, u_{4}\right)=(-1)^{i}\left(u_{1} u_{3}-u_{2} u_{4}\right) .
$$

In this case, we check that (1.20) is

$$
\begin{gathered}
\text { for } J=\{1,2,3\}, \quad\left(\exists j \in\{1,2,3\}, \partial_{j} f_{4}\left(u_{1}^{*}, \ldots, u_{4}^{*}\right) \neq 0\right) \Leftrightarrow\left(\left(u_{1}^{*}, u_{3}^{*}, u_{4}^{*}\right) \neq(0,0,0)\right), \\
\text { for } J=\{1,2\}, \quad\left(\exists j \in\{1,2\}, \partial_{j} f_{3}\left(u_{1}^{*}, \ldots, u_{4}^{*}\right) \neq 0\right) \Leftrightarrow\left(\left(u_{3}^{*}, u_{4}^{*}\right) \neq(0,0)\right) \\
\text { for } J=\{1\}, \quad\left(\partial_{1} f_{2}\left(u_{1}^{*}, \ldots, u_{4}^{*}\right) \neq 0\right) \Leftrightarrow\left(u_{3}^{*} \neq 0\right) .
\end{gathered}
$$

Thus, Theorem 1.7 recovers the result of ([24], Thm. 3.2) except for the case $J=\{1,2,3\}$ and $\left(u_{1}^{*}, u_{3}^{*}, u_{4}^{*}\right)=$ $(0,0,0)$ that the proof of the present article does not treat (see Example 7.1 for more details about the strategy of $[24])$.

We will only prove Theorem 1.7 under the assumption $m \leq n-2$. The other cases are an easy adaptation.

\subsection{Bibliographical comments}

In this section, we recall some known results about the null-controllability of linear and semilinear parabolic systems with Neumann boundary conditions to put in perspective the statement and the proof strategy of Theorem 1.7.

\subsubsection{Linear results}

Let $k, l \in \mathbb{N}^{*}$. We denote by $\mathcal{M}_{k}(\mathbb{R})\left(\right.$ respectively $\left.\mathcal{M}_{k, l}(\mathbb{R})\right)$ the algebra of matrices with $k$ lines and $k$ columns (respectively the algebra of matrices with $k$ lines and $l$ columns) with entries in $\mathbb{R}$. For $M \in \mathcal{M}_{k}(\mathbb{R}), \operatorname{Sp}(M)$ is the set of complex eigenvalues of $M: \operatorname{Sp}(M):=\left\{\lambda \in \mathbb{C} ; \exists X \in \mathbb{C}^{k} \backslash\{0\}, M X=\lambda X\right\}$.

Since the pioneer works of Gilles Lebeau, Luc Robbiano in 1995 (see [20, 26] and the survey [25]) and Andrei Fursikov, Oleg Imanuvilov in 1996 (see [13, 16]) about the null-controllability of the heat equation, the control of coupled parabolic systems has been a challenging issue in the last twenty years. For instance, in [1], the authors identify necessary and sufficient conditions for the null-controllability of linear parabolic systems of the following form

$$
\begin{cases}\partial_{t} Z-\Gamma \Delta Z=A Z+B H 1_{\omega} & \text { in }(0, T) \times \Omega \\ \frac{\partial Z}{\partial \nu}=0 & \text { on }(0, T) \times \partial \Omega \\ Z(0, .)=Z_{0} & \text { in } \Omega\end{cases}
$$

where $\Gamma$ a diagonalizable matrix of $\mathcal{M}_{k}(\mathbb{R})$ with $\operatorname{Sp}(\Gamma) \subset(0,+\infty), A \in \mathcal{M}_{k}(\mathbb{R}), B \in \mathcal{M}_{k, l}(\mathbb{R})$. In general, the rank of $B$ is less that $k$, so that the controllability of the full system (1.22) depends strongly on the coupling present in the system. 
Inspired by the works $[17,18,27]$, a byproduct of this article is a new null-controllability result, for cascade cross-diffusion systems of arbitrary size (see Sect. 3, Thm. 3.1).

For a recent survey on the null-controllability of linear parabolic systems, see [2] and references therein.

\subsubsection{Semilinear results}

For semilinear parabolic systems

$$
\begin{cases}\partial_{t} Z-\Gamma \Delta Z=G(Z)+B H 1_{\omega} & \text { in }(0, T) \times \Omega, \\ \frac{\partial Z}{\partial \nu}=0 & \text { on }(0, T) \times \partial \Omega, \\ Z(0, .)=Z_{0} & \text { in } \Omega,\end{cases}
$$

with $G \in C^{\infty}\left(\mathbb{R}^{k} ; \mathbb{R}^{k}\right)$ such that $G(0)=0$, the usual strategy consists in deducing a local null-controllability result for (1.23) from a (global) null-controllability result for the linearized system around $(Z, H)=(0,0)$. This linear system takes the form (1.22) with the matrix $A$ defined as follows: $a_{i, j}=\partial_{j} g_{i}(0)(1 \leq i, j \leq k)$. In this paper, we use the powerful source term method, introduced by Yuning Liu, Takéo Takahashi and Marius Tucsnak in [28]. One of the main advantage of the method is to deduce the local null-controllability for (1.23) from the null-controllability of only one linear system (1.22).

In this article, we adapt the source term method in a $L^{\infty}$-context in the following way.

- The source term method in $L^{2}$ enables to prove a strong observability inequality (see Cor. 4.4). This estimate looks like a global Carleman estimate (see for example [13], Lem. 1.3), whereas the method to get it is very different.

- By using the Penalized Hilbert Uniqueness Method, introduced by Viorel Barbu in [3], we construct $L^{\infty}$-controls (see Thm. 5.1).

- We use once more the source term method in $L^{\infty}$ (see Prop. 5.3).

- We conclude by an appropriate inverse mapping theorem (see Sect. 6).

For other results using the source term method, see for instance [5, 14, 29].

\section{AN ADEQUATE CHANGE OF VARIABLES AND LINEARIZATION}

\subsection{Change of variables - cross diffusion system}

The goal of this section is to transform the controlled system (NL-U) (see Sect. 1.2) satisfied by $U$ into another system of cascade type for which we better understand the controllability properties. Roughly speaking, for $1 \leq i \leq m$, the component $u_{i}$ is easy to control thanks to the localized control term $h_{i} 1_{\omega}$. Thus, the challenge is to understand how the reaction term $f_{i}(U)$ (see (1.6)) acts on the component $u_{i}$ for $m+1 \leq i \leq n$.

We multiply the $(m+1)$ th equation (respectively the $(m+2)$ th equation) of (NL-U) by

$$
\left(\left(\beta_{m+1}-\alpha_{m+1}\right)\left(d_{m+1}-d_{m+2}\right)\right)^{-1}\left(\text { respectively }\left(\left(\beta_{m+2}-\alpha_{m+2}\right)\left(d_{m+2}-d_{m+1}\right)\right)^{-1}\right),
$$

and we sum:

$$
\partial_{t} v_{m+2}-d_{m+2} \Delta v_{m+2}=\frac{\Delta u_{m+1}}{\beta_{m+1}-\alpha_{m+1}}
$$

where

$$
v_{m+2}=\frac{u_{m+1}}{\left(\beta_{m+1}-\alpha_{m+1}\right)\left(d_{m+1}-d_{m+2}\right)}+\frac{u_{m+2}}{\left(\beta_{m+2}-\alpha_{m+2}\right)\left(d_{m+2}-d_{m+1}\right)}
$$


Roughly speaking, this linear combination enables to "kill" the reaction-term and to create a coupling term of second order.

By iterating this strategy, we construct a linear transformation $V=P U$ such that $u_{m+1}$ acts on $v_{m+2}$, $v_{m+2}$ acts on $v_{m+3}, \ldots, v_{n-1}$ acts on $v_{n}$ through cross diffusion terms. Moreover, we transform the problem of controllability for $U$ to $U^{*}$ into a null-controllability problem for

$$
Z:=P\left(U-U^{*}\right)
$$

where $P$ is the invertible triangular matrix defined by:

$$
P:=\left(\begin{array}{c|c}
I_{m} & (0) \\
\hline(0) & *
\end{array}\right)
$$

with

$$
\forall k, l \geq m+1, P_{k l}:=\left\{\begin{array}{cl}
\left(\left(\beta_{l}-\alpha_{l}\right) \underset{\substack{m+1 \leq r \leq k \\
r \neq l}}{\left.\prod_{\substack{n \\
0}}\left(d_{l}-d_{r}\right)\right)^{-1}}\right. & \text { if } k \geq l, \\
\text { if } k<l,
\end{array}\right.
$$

with the convention $\prod_{\emptyset}=1$.

We introduce the notations:

$$
G(Z):=\left(g_{1}(Z), \ldots, g_{m+1}(Z), 0 \ldots, 0\right)^{\operatorname{tr}}
$$

with

$$
g_{i}(Z):=f_{i}\left(P^{-1} Z+U^{*}\right)(1 \leq i \leq m), g_{m+1}(Z):=\frac{f_{i}\left(P^{-1} Z+U^{*}\right)}{\beta_{m+1}-\alpha_{m+1}}
$$

and

$$
D_{J}:=\left(\begin{array}{c|c}
\operatorname{diag}\left(d_{1}, \ldots, d_{m}\right) & (0) \\
\hline(0) & D_{\sharp}
\end{array}\right), D_{\sharp}:=\left(\begin{array}{ccccc}
d_{m+1} & 0 & \ldots & \ldots & 0 \\
1 & d_{m+2} & \ddots & \ddots & \vdots \\
0 & \ddots & \ddots & \ddots & \vdots \\
\vdots & \ddots & \ddots & \ddots & 0 \\
0 & \ldots & 0 & 1 & d_{n}
\end{array}\right) .
$$

Proposition 2.1. Let $U_{0} \in L^{\infty}(\Omega)^{n}, H^{J} \in L^{\infty}\left(Q_{T}\right)^{m}$. Then, $U$ is a solution of (NL-U) if and only if $Z$ satisfies

$$
\begin{cases}\partial_{t} Z-D_{J} \Delta Z=G(Z)+H^{J} 1_{\omega} & \text { in }(0, T) \times \Omega, \\ \frac{\partial Z}{\partial \nu}=0 & \text { on }(0, T) \times \partial \Omega \\ Z(0, .)=Z_{0} & \text { in } \Omega\end{cases}
$$

The proof of Proposition 2.1 is done in Appendix A.4.1. 
Remark 2.2. The concept of solution for (NL-Z) is an easy adaptation of the notion of solution for (NL-U) given in Definition 1.1.

Let $p \in[1,+\infty]$. We introduce the following subspace of $L^{p}(\Omega)^{n}$ :

$$
L_{\mathrm{inv}}^{p}:=\left\{Z_{0} \in L^{p}(\Omega)^{n} ; \forall m+2 \leq i \leq n, \int_{\Omega} z_{i, 0}(x) \mathrm{d} x=0\right\} .
$$

Theorem 1.7 is equivalent to the following local null-controllability theorem for (NL-Z).

Theorem 2.3. Under Assumptions 1.4 and 1.6, the system (NL-Z) is locally null-controllable, i.e., there exists $r>0$ such that for every $Z_{0} \in L_{\mathrm{inv}}^{\infty}$ verifying $\left\|Z_{0}\right\|_{L^{\infty}(\Omega)^{n}} \leq r$, there exists $H^{J} \in L^{\infty}\left(Q_{T}\right)^{m}$ such that the solution $Z$ of (NL-Z) satisfies $Z(T,)=$.0 .

The equivalence between Theorems 1.7 and 2.3 comes from Proposition 2.1 and the following equivalence

$$
Z_{0} \in L_{\mathrm{inv}}^{\infty} \Leftrightarrow U_{0} \text { satisfies }(1.16) \Leftrightarrow U_{0} \text { satisfies (1.18) (Rem. 1.5). }
$$

The proof of (2.7) is done in Appendix A.4.2.

From now, we will focus on the proof of Theorem 2.3.

\subsection{Linearization}

The linearized system of (NL-Z) around $(0,0)$ is

$$
\begin{cases}\partial_{t} Z-D_{J} \Delta Z=A_{J} Z+H^{J} 1_{\omega} & \text { in }(0, T) \times \Omega, \\ \frac{\partial Z}{\partial \nu}=0 & \text { on }(0, T) \times \partial \Omega \\ Z(0, .)=Z_{0} & \text { in } \Omega,\end{cases}
$$

where

$$
A_{J}=\left(a_{i k}\right)_{1 \leq i, k \leq n}, \quad a_{i k}=\left\{\begin{array}{cl}
\partial_{k} g_{i}(0, \ldots, 0) & \text { if } 1 \leq i \leq m+1 \\
0 & \text { if } m+2 \leq i \leq n
\end{array}\right.
$$

By Assumption 1.6, (2.1) and (2.4), we have

$$
a_{m+1, m} \neq 0 \text {. }
$$

Roughly speaking, we summarize the expected controllability properties in the following diagram:

$$
\begin{aligned}
& h_{1} \stackrel{\text { controls }}{\longrightarrow} z_{1}, h_{2} \stackrel{\text { controls }}{\longrightarrow} z_{2}, \ldots, h_{m-1} \stackrel{\text { controls }}{\longrightarrow} z_{m-1}, \\
& h_{m} \stackrel{\text { controls }}{\longrightarrow} z_{m} \underset{a_{m+1 m} z_{m}}{\stackrel{\text { controls }}{\longrightarrow}} z_{m+1} \stackrel{\text { controls }}{\Delta z_{m+1}} z_{m+2} \frac{\text { controls }}{\Delta z_{m+2}} \ldots \frac{\text { controls }}{\Delta z_{n-1}} z_{n} .
\end{aligned}
$$

\section{LiNEAR NULL-CONTROLLABILITY UNDER CONSTRAINTS IN $L^{2}$}

The main result of this section, stated in the following theorem, is the null-controllability in $L_{\text {inv }}^{2}$ for the linear system (L-Z) (see Sect. 2.2).

Theorem 3.1. The system (L-Z) is null-controllable in $L_{\mathrm{inv}}^{2}$. More precisely, there exists $C>0$ such that for every $T>0$ and $Z_{0} \in L_{\text {inv }}^{2}$, there exists a control $H^{J} \in L^{2}\left(Q_{T}\right)^{m}$ verifying

$$
\left\|H^{J}\right\|_{L^{2}\left(Q_{T}\right)^{m}} \leq C_{T}\left\|Z_{0}\right\|_{L^{2}(\Omega)^{n}}, \text { where } C_{T}=C e^{C / T},
$$


and such that the solution $Z \in W_{T}^{n}$ of (L-Z) satisfies $Z(T,)=$.0 .

The goal of the next two subsections is to prove Theorem 3.1. The proof is based on the Lebeau-Robbiano's method, introduced for the first time to prove the null-controllability of the heat equation (see [26]). First, it consists in establishing a null-controllability result in finite dimensional subspaces of $L_{\mathrm{inv}}^{2}$ with a precise estimate of the cost of the control (see Prop. 3.2). This first step is based on two main results: the spectral inequality for eigenfunctions of the Neumann-Laplace operator (see Lem. 3.4) and precise observability estimates of linear finite dimensional systems associated to the adjoint system of (L-Z) (see Lem. 3.5). Secondly, we conclude by a time-splitting procedure: the control $H^{J}$ is built as a sequence of active controls and passive controls. The passive mode allows to take advantage of the natural parabolic exponential decay of the $L^{2}$ norm of the solution. This decay enables to compensate the cost of the control which steers the low frequencies to 0 (see Sect. 3.2).

We must be careful with the dependence on the constants appearing in the estimates with respect to $T$ (when $T$ is small). That is why, from now and until the end of the article, we assume that

$$
T \in(0,1)
$$

Unless otherwise specified, we denote by $C$ various positive constants varying from line to line.

\subsection{A null-controllability result for the low frequencies}

The unbounded operator on $L^{2}(\Omega):\left(-\Delta, H_{N e}^{2}(\Omega)\right)$, where $H_{N e}^{2}(\Omega)$ is defined in (A.4) (see Appendix A.1.2) is self-adjoint and has compact resolvent. Thus, we introduce the orthonormal basis $\left(e_{k}\right)_{k \geq 0}$ of $L^{2}(\Omega)$ of eigenfunctions associated to the increasing sequence of eigenvalues $\left(\lambda_{k}\right)_{k \geq 0}$ of the Laplacian operator, i.e., we have $-\Delta e_{k}=\lambda_{k} e_{k}$ and $\left(e_{k}, e_{l}\right)_{L^{2}(\Omega)}=\delta_{k, l}$. For $\lambda>0$, we define the finite dimensional space $E_{\lambda}=$ $\left\{\sum_{\lambda_{k} \leq \lambda} c_{k} e_{k} ; c_{k} \in \mathbb{R}^{n}\right\} \subset L^{2}(\Omega)^{n}$ and the orthogonal projection $\Pi_{E_{\lambda}}$ onto $E_{\lambda}$ in $L^{2}(\Omega)^{n}$.

The goal of this section is to prove the following null-controllability result in a finite dimensional subspace of $L_{\mathrm{inv}}^{2}$.

Proposition 3.2. There exist $C>0, p_{1} \in \mathbb{N}$ such that for every $\tau \in(0, T), \lambda>0, Z_{0} \in E_{\lambda} \cap L_{\text {inv }}^{2}$, there exists a control function $H^{J} \in L^{2}\left(Q_{\tau}\right)$ verifying

$$
\left\|H^{J}\right\|_{L^{2}\left(Q_{\tau}\right)^{m}}^{2} \leq \frac{C}{\tau^{p_{1}}} e^{C \sqrt{\lambda}}\left\|Z_{0}\right\|_{L^{2}(\Omega)^{n}}^{2},
$$

such that the solution $Z$ of

$$
\begin{cases}\partial_{t} Z-D_{J} \Delta Z=A_{J} Z+H^{J} 1_{\omega} & \text { in }(0, \tau) \times \Omega \\ \frac{\partial Z}{\partial \nu}=0 & \text { on }(0, \tau) \times \partial \Omega \\ Z(0, .)=Z_{0} \in E_{\lambda} & \text { in } \Omega\end{cases}
$$

satisfies $Z(\tau,)=$.0 .

From Proposition 3.2, for every $\tau, \lambda>0$ and $Z_{0} \in E_{\lambda} \cap L_{\text {inv }}^{2}$, we introduce the notation:

$$
H_{\lambda}\left(Z_{0}, 0, \tau\right):=H^{J}
$$

such that the solution $Z$ of (3.4) satisfies $Z(\tau,)=$.0 and $H^{J}$ is the minimal-norm element of $L^{2}\left(Q_{\tau}\right)^{m}$ satisfying the estimate (3.3). In other words, $H^{J}$ is the projection of 0 in the nonempty closed convex set of controls satisfying (3.3) and driving the solution $Z$ of (3.4) in time $\tau-0$. 
By the Hilbert Uniqueness Method (see [8], Thm. 2.44), in order to prove Proposition 3.2, we need to prove an observability inequality for the solution of the adjoint system of (3.4).

Proposition 3.3. There exist $C>0, p_{1} \in \mathbb{N}$ such that for every $\tau \in(0, T), \lambda>0$ and $\varphi_{\tau} \in E_{\lambda} \cap L_{\text {inv }}^{2}$, the solution $\varphi$ of

$$
\begin{cases}-\partial_{t} \varphi-D_{J}^{\operatorname{tr}} \Delta \varphi=A_{J}^{\operatorname{tr}} \varphi & \text { in }(0, \tau) \times \Omega \\ \frac{\partial \varphi}{\partial \nu}=0 & \text { on }(0, \tau) \times \partial \Omega \\ \varphi(\tau, .)=\varphi_{\tau} & \text { in } \Omega\end{cases}
$$

satisfies

$$
\|\varphi(0, .)\|_{L^{2}(\Omega)^{n}}^{2} \leq \frac{C}{\tau^{p_{1}}} e^{C \sqrt{\lambda}} \sum_{i=1}^{m} \int_{0}^{\tau} \int_{\omega}\left|\varphi_{i}(t, x)\right|^{2} \mathrm{~d} x \mathrm{~d} t .
$$

Proof. The proof is inspired by ([27], Sect. 3).

Let $\tau, \lambda>0$ and $\varphi_{\tau} \in E_{\lambda} \cap L_{\text {inv }}^{2}$. We have:

$$
\varphi_{\tau}(x)=\sum_{\lambda_{k} \leq \lambda} \varphi_{k}^{\tau} e_{k}(x)
$$

with $\varphi_{k}^{\tau} \in F_{k}$ where $F_{0}:=\mathbb{R}^{m+1} \times\{0\}^{n-m-1}$ because $\varphi_{\tau} \in L_{\text {inv }}^{2}$ and $F_{k}:=\mathbb{R}^{n}$ for $k \geq 1$.

Then, the solution $\varphi$ of (3.6) is

$$
\forall(t, x) \in(0, \tau) \times \Omega, \varphi(t, x)=\sum_{\lambda_{k} \leq \lambda} \varphi_{k}(t) e_{k}(x),
$$

where $\varphi_{k}$ is the unique solution of the ordinary differential system

$$
\left\{\begin{array}{l}
-\varphi_{k}^{\prime}+\lambda_{k} D_{J}^{\operatorname{tr}} \varphi_{k}=A_{J}^{\operatorname{tr}} \varphi_{k}, \text { in }(0, \tau) \\
\varphi_{k}(\tau)=\varphi_{k}^{\tau}
\end{array}\right.
$$

We recall the spectral inequality for eigenfunctions of the Neumann-Laplace operator.

Lemma 3.4. ([20], Thm. 14.6)

There exists $C>0$ such that for every sequence $\left(a_{k}\right)_{k \geq 0} \subset \mathbb{C}^{\mathbb{N}}$ and for every $\lambda>0$, we have:

$$
\sum_{\lambda_{k} \leq \lambda}\left|a_{k}\right|^{2}=\int_{\Omega}\left|\sum_{\lambda_{k} \leq \lambda} a_{k} e_{k}(x)\right|^{2} \mathrm{~d} x \leq C e^{C \sqrt{\lambda}} \int_{\omega}\left|\sum_{\lambda_{k} \leq \lambda} a_{k} e_{k}(x)\right|^{2} \mathrm{~d} x .
$$

By using (3.10) for $a_{k}=\varphi_{k, i}(t)$ with $1 \leq i \leq m$ and by summing on $1 \leq i \leq m$, we obtain that there exists $C>0$ such that

$$
\sum_{\lambda_{k} \leq \lambda} \sum_{i=1}^{m}\left|\varphi_{k, i}(t)\right|^{2} \leq C e^{C \sqrt{\lambda}} \sum_{i=1}^{m} \int_{\omega}\left|\sum_{\lambda_{k} \leq \lambda} \varphi_{k, i}(t) e_{k}(x)\right|^{2} \mathrm{~d} x .
$$


By integrating with respect to the time variable between 0 and $\tau$ the inequality (3.11), we obtain

$$
\int_{0}^{\tau} \sum_{\lambda_{k} \leq \lambda} \sum_{i=1}^{m}\left|\varphi_{k, i}(t)\right|^{2} \mathrm{~d} t \leq C e^{C \sqrt{\lambda}} \sum_{i=1}^{m} \int_{0}^{\tau} \int_{\omega}\left|\sum_{\lambda_{k} \leq \lambda} \varphi_{k, i}(t) e_{k}(x)\right|^{2} \mathrm{~d} x \mathrm{~d} t .
$$

Moreover, we have the following lemma whose proof is postponed in Appendix A.5 (see also [32]).

Lemma 3.5. There exist $C>0,\left(p_{1}, p_{2}\right) \in \mathbb{N}^{2}$ such that for every $\tau \in(0,1), k \in \mathbb{N}, \varphi_{k}^{\tau} \in F_{k}$, the solution $\varphi_{k}$ of (3.9) satisfies

$$
\left\|\varphi_{k}(0)\right\|^{2} \leq C\left(1+\frac{1}{\tau^{p_{1}}}+\lambda_{k}^{p_{2}}\right) \sum_{i=1}^{m} \int_{0}^{\tau}\left|\varphi_{k, i}(t)\right|^{2} \mathrm{~d} t .
$$

By using (3.12), (3.13), we deduce that

$$
\begin{aligned}
\sum_{\lambda_{k} \leq \lambda}\left\|\varphi_{k}(0)\right\|^{2} & \leq \sum_{\lambda_{k} \leq \lambda} \frac{C}{\tau^{p_{1}}}\left(1+\lambda_{k}^{p_{2}}\right) \sum_{i=1}^{m} \int_{0}^{\tau}\left|\varphi_{k, i}(t)\right|^{2} \mathrm{~d} t \\
& \leq \frac{C}{\tau^{p_{1}}} e^{C \sqrt{\lambda}} \sum_{i=1}^{m} \int_{0}^{\tau} \int_{\omega}\left|\sum_{\lambda_{k} \leq \lambda} \varphi_{k, i}(t) e_{k}(x)\right|^{2} \mathrm{~d} x \mathrm{~d} t .
\end{aligned}
$$

By using (3.8), we deduce (3.7) from (3.14).

\subsection{The Lebeau-Robbiano's method}

The goal of this section is to prove Theorem 3.1.

Proof. The proof is inspired by ([25], Sect. 6.2) (see also [26], Fin de la preuve du Thm. 1). The constants $C, C^{\prime}$ will increase from line to line.

We split the interval $[0, T]=\cup_{k \in \mathbb{N}}\left[a_{k}, a_{k+1}\right]$ with $a_{0}=0, a_{k+1}=a_{k}+2 T_{k}$ and $T_{k}=\kappa T / 2^{k}$ for $k \in \mathbb{N}$ and the constant $\kappa$ is chosen such that $2 \sum_{k=0}^{+\infty} T_{k}=T$. We also define $\mu_{k}=M 2^{2 k}$ for $M>0$ sufficiently large which will be defined later and for $k \in \mathbb{N}$. Then, we define the control $H^{J}$ in the following way:

- if $t \in\left(a_{k}, a_{k}+T_{k}\right), H^{J}=H_{\mu_{k}}\left(\Pi_{E_{\mu_{k}}} Z\left(a_{k},.\right), a_{k}, T_{k}\right)$ (see the notation (3.5)) and $Z(t,)=.S(t-$ $\left.a_{k}\right) Z\left(a_{k},.\right)+\int_{a_{k}}^{t} S(t-s) H^{J}(s,.) \mathrm{d} s$,

- if $t \in\left(a_{k}+T_{k}, a_{k+1}\right), H^{J}=0$ and $Z(t,)=.S\left(t-a_{k}-T_{k}\right) Z\left(a_{k}+T_{k},.\right)$,

where $S(t)$ denotes the semigroup of the parabolic system: $S(t)=e^{t\left(D_{J} \Delta+A_{J}\right)}$. In particular, by (A.2) and (1.11), $\|S(t)\|_{\mathcal{L}\left(L^{2}(\Omega)^{n}\right)} \leq C$.

By (3.3), the choice of $H^{J}$ during the interval time $\left[a_{k}, a_{k}+T_{k}\right]$ implies

$$
\begin{aligned}
\left\|Z\left(a_{k}+T_{k}, .\right)\right\|_{L^{2}(\Omega)^{n}}^{2} & \leq\left(C+C\left(\kappa 2^{-k} T\right)^{-p_{1}} e^{C \sqrt{M} 2^{k}}\right)\left\|Z\left(a_{k}, .\right)\right\|_{L^{2}(\Omega)^{n}}^{2} \\
& \leq \frac{C}{T^{p_{1}}} e^{C \sqrt{M} 2^{k}}\left\|Z\left(a_{k}, .\right)\right\|_{L^{2}(\Omega)^{n}}^{2} .
\end{aligned}
$$

During the passive period of the control, $t \in\left[a_{k}+T_{k}, a_{k+1}\right]$, the solution exponentially decreases:

$$
\left\|Z\left(a_{k+1}, .\right)\right\|_{L^{2}(\Omega)^{n}}^{2} \leq C^{\prime} e^{-C^{\prime} M 2^{2 k} T_{k}}\left\|Z\left(a_{k}+T_{k}, .\right)\right\|_{L^{2}(\Omega)^{n}}^{2} .
$$


Thus, by using $2^{2 k} T_{k}=\kappa 2^{k} T$, (3.15) and (3.16), we have

$$
\left\|Z\left(a_{k+1}, .\right)\right\|_{L^{2}(\Omega)^{n}}^{2} \leq \frac{C}{T^{p_{1}}} e^{C \sqrt{M} 2^{k}-C^{\prime} M 2^{k} T}\left\|Z\left(a_{k}, .\right)\right\|_{L^{2}(\Omega)^{n}}^{2},
$$

and consequently,

$$
\begin{aligned}
\left\|Z\left(a_{k+1}, .\right)\right\|_{L^{2}(\Omega)^{n}}^{2} & \leq\left(\frac{C}{T^{p_{1}}}\right)^{k+1} e^{\sum_{j=0}^{k}\left(C \sqrt{M} 2^{j}-C^{\prime} M T 2^{j}\right)}\left\|Z_{0}\right\|_{L^{2}(\Omega)^{n}}^{2} \\
& \leq e^{C / T+\left(C \sqrt{M}-C^{\prime} M T\right) 2^{k+1}}\left\|Z_{0}\right\|_{L^{2}(\Omega)^{n}}^{2} .
\end{aligned}
$$

By taking $M$ such that $C \sqrt{M}-C^{\prime} M T<0$, for instance $M \geq 2\left(C / C^{\prime} T\right)^{2}$, we conclude by (3.17) that we have $\lim _{k \rightarrow+\infty}\left\|Z\left(a_{k},.\right)\right\|=0$, i.e., $Z(T,)=$.0 because $t \mapsto Z(t,.) \in C\left([0, T] ; L^{2}(\Omega)^{n}\right)$ because $H^{J} \in L^{2}\left(Q_{T}\right)^{m}$ (see Prop. A.2 and (1.11)) as we will show now.

We have $\left\|H^{J}\right\|_{L^{2}\left(Q_{T}\right)^{m}}^{2}=\sum_{k=0}^{+\infty}\left\|H^{J}\right\|_{L^{2}\left(\left(a_{k}, a_{k}+T_{k}\right) \times \Omega\right)^{m}}^{2}$. Then, by using the estimate (3.3) of the control on each time interval $\left(a_{k}, a_{k}+T_{k}\right)$ and the estimate (3.17), we get:

$$
\begin{aligned}
\left\|H^{J}\right\|_{L^{2}\left(Q_{T}\right)^{m}}^{2} & \leq\left(C T_{0}^{-p_{1}} e^{C \sqrt{M}}+\sum_{k \geq 1} C T_{k}^{-p_{1}} e^{C \sqrt{M} 2^{k}} e^{C / T+\left(C \sqrt{M}-C^{\prime} M T\right) 2^{k}}\right)\left\|Z_{0}\right\|_{L^{2}(\Omega)^{n}}^{2} \\
& \leq\left(C T^{-p_{1}} e^{C \sqrt{M}}+\sum_{k \geq 1} C\left(2^{k} T^{-1}\right)^{p_{1}} e^{C / T} e^{\left(2 C \sqrt{M}-C^{\prime} M T\right) 2^{k}}\right)\left\|Z_{0}\right\|_{L^{2}(\Omega)^{n}}^{2} .
\end{aligned}
$$

By taking $M$ such that $2 C \sqrt{M}-C^{\prime} M T<0$, for instance $M=8\left(C / C^{\prime} T\right)^{2} \Rightarrow C \sqrt{M}-C^{\prime} M T / 2=-C^{\prime \prime} / T$ with $C^{\prime \prime}>0$, we deduce from (3.18) that $H^{J} \in L^{2}\left(Q_{T}\right)^{m}$ and

$$
\left\|H^{J}\right\|_{L^{2}\left(Q_{T}\right)^{m}}^{2} \leq C e^{C / T} \int_{0}^{+\infty}\left(\frac{\sigma}{T}\right)^{p_{1}} e^{-C^{\prime \prime} \frac{\sigma}{T}} \mathrm{~d} \sigma\left\|Z_{0}\right\|_{L^{2}(\Omega)^{n}}^{2} \leq C e^{C / T}\left\|Z_{0}\right\|_{L^{2}(\Omega)^{n}}^{2},
$$

which concludes the proof of Theorem 3.1.

\section{THE SOURCE TERM METHOD IN $L^{2}$}

We use the source term method, introduced by Yuning Liu, Takéo Takahashi and Marius Tucsnak in ([28], Prop. 2.3) to deduce a local null-controllability result for a nonlinear system from the null-controllability result for only one linear system (and an estimate of the cost of the control) (see also [5]).

By Theorem 3.1, we have an estimate for the control cost in $L^{2}$, then we fix $M>0$ such that $C_{T} \leq M e^{M / T}$. Let $q \in(1, \sqrt{2})$ and $p>q^{2} /\left(2-q^{2}\right)$. We define the weights

$$
\begin{gathered}
\rho_{0}(t):=M^{-p} \exp \left(-\frac{M p}{(q-1)(T-t)}\right), \\
\rho_{\mathcal{S}}(t)=M^{-1-p} \exp \left(-\frac{(1+p) q^{2} M}{(q-1)(T-t)}\right) .
\end{gathered}
$$


Remark 4.1. The assumption $p>q^{2} /\left(2-q^{2}\right) \Leftrightarrow 2 p>(1+p) q^{2}$ implies

$$
\rho_{0}^{2} / \rho_{\mathcal{S}} \in C([0, T])
$$

which will be useful for the estimate of the polynomial nonlinearity (see Sect. 6).

Let $r \in\{2,+\infty\}$. For $S \in L^{r}\left((0, T) ; L_{\text {inv }}^{r}\right), H^{J} \in L^{r}\left((0, T) ; L^{r}(\Omega)^{m}\right), Z_{0} \in L_{\text {inv }}^{r}$, we introduce the following system:

$$
\begin{cases}\partial_{t} Z-D_{J} \Delta Z=A_{J} Z+S+H^{J} 1_{\omega} & \text { in }(0, T) \times \Omega \\ \frac{\partial Z}{\partial \nu}=0 & \text { on }(0, T) \times \partial \Omega \\ Z(0, .)=Z_{0} & \text { in } \Omega\end{cases}
$$

Then, we define associated spaces for the source term, the state and the control

$$
\begin{aligned}
& \mathcal{S}_{r}:=\left\{S \in L^{r}\left((0, T) ; L_{\mathrm{inv}}^{r}\right) ; \frac{S}{\rho_{\mathcal{S}}} \in L^{r}\left((0, T) ; L_{\mathrm{inv}}^{r}\right)\right\}, \\
& \mathcal{Z}_{r}:=\left\{Z \in L^{r}\left((0, T) ; L_{\mathrm{inv}}^{r}\right) ; \frac{Z}{\rho_{0}} \in L^{r}\left((0, T) ; L_{\mathrm{inv}}^{r}\right)\right\}, \\
& \mathcal{H}_{r}:=\left\{H^{J} \in L^{r}\left((0, T) ; L^{r}(\Omega)^{m}\right) ; \frac{H^{J}}{\rho_{0}} \in L^{r}\left((0, T) ; L^{r}(\Omega)^{m}\right)\right\} .
\end{aligned}
$$

Remark 4.2. From the behaviors near $t=T$ of $\rho_{\mathcal{S}}$ and $\rho_{0}$, we deduce that each element of $\mathcal{S}_{r}, \mathcal{Z}_{r}, \mathcal{H}_{r}$ vanishes at $t=T$.

From the abstract result: ([28], Prop. 2.3), we deduce the null-controllability for (L+S-Z) in $L_{\mathrm{inv}}^{2}$.

Proposition 4.3. For every $S \in \mathcal{S}_{2}$ and $Z_{0} \in L_{\text {inv }}^{2}$, there exists $H^{J} \in \mathcal{H}_{2}$, such that the solution $Z$ of $(\mathrm{L}+\mathrm{S}-\mathrm{Z})$ satisfies $Z \in \mathcal{Z}_{2}$. Furthermore, there exists $C>0$, not depending on $S$ and $Z_{0}$, such that

$$
\left\|Z / \rho_{0}\right\|_{C\left([0, T] ; L^{2}(\Omega)^{n}\right)}+\left\|H^{J}\right\|_{\mathcal{H}_{2}} \leq C_{T}\left(\left\|Z_{0}\right\|_{L^{2}(\Omega)^{n}}+\|S\|_{\mathcal{S}_{2}}\right),
$$

where $C_{T}=C e^{C / T}$. In particular, since $\rho_{0}$ is a continuous function satisfying $\rho_{0}(T)=0$, the above relation (4.7) yields $Z(T,)=$.0 .

For the sake of completeness, the proof of Proposition 4.3 is in Appendix A.6 (see Prop. A.11 applied with $r=2$ ).

Now, we will deduce an observability estimate for the adjoint system:

$$
\begin{cases}-\partial_{t} \varphi-D_{J}^{\operatorname{tr}} \Delta \varphi=A_{J}^{\operatorname{tr}} \varphi & \text { in }(0, T) \times \Omega \\ \frac{\partial \varphi}{\partial \nu}=0 & \text { on }(0, T) \times \partial \Omega \\ \varphi(T, .)=\varphi_{T} & \text { in } \Omega .\end{cases}
$$

We have the following result which is an adaptation of ([28], Cor. 2.6) or ([19], Thm. 4.1) (see Appendix A.7 for a complete proof).

Corollary 4.4. There exists $C>0$ such that for every $\varphi_{T} \in L_{\text {inv }}^{2}$, the solution of (4.8) satisfies:

$$
\|\varphi(0, .)\|_{L^{2}(\Omega)^{n}}^{2}+\int_{0}^{T} \int_{\Omega}\left|\rho_{\mathcal{S}}(t) \varphi(t, x)\right|^{2} \leq C_{T}\left(\sum_{i=1}^{m} \int_{0}^{T} \int_{\omega}\left|\rho_{0}(t) \varphi_{i}(t, x)\right|^{2}\right),
$$

where $C_{T}=C e^{C / T}$. 
In the next section, we take advantage of the strong observability estimate (4.9) to get more regularity in $L^{p}$-sense for the control $H^{J}$.

\section{Construction of $L^{\infty}$-CONTROLS And The SOURCE TERM METHOD IN $L^{\infty}$}

\subsection{The Penalized Hilbert Uniqueness Method to build $L^{\infty}$-controls}

The goal of this section is to prove a null-controllability result in $L^{\infty}$ with an estimate of the cost of the control.

Theorem 5.1. There exists $C>0$ such that for every $T>0, Z_{0} \in L_{\text {inv }}^{2}$, there exists a control $H^{J} \in L^{\infty}\left(Q_{T}\right)^{m}$ verifying

$$
\left\|H^{J}\right\|_{L^{\infty}\left(Q_{T}\right)^{m}} \leq C_{T}\left\|Z_{0}\right\|_{L^{2}(\Omega)^{n}} \text {, where } C_{T}=C e^{C / T} .
$$

and such that the solution $Z$ of (L-Z) (see Sect. 2.2) satisfies $Z(T,)=$.0 .

From now and until the end of the section, we will denote by $C_{T}$ various positive constants which can change from line to line and such that $C_{T} \leq C e^{C / T}$.

In the next four parts, we perform the usual Penalized Hilbert Uniqueness Method, introduced for the first time by Viorel Barbu in [3]. The idea is the following one: it is a well-known fact that the optimal control $H^{J} \in L^{2}((0, T) \times \Omega)^{m}$, i.e., the minimal-norm element in $L^{2}$, which steers the solution $Z$ of (L-Z) to 0 in time $T$ can be expressed as a function of a solution of the adjoint system (4.8) (see [8], Sect. 1.4 for more details in the context of linear finite dimensional controlled systems). By using the strong observability inequality (4.9), we will use this link by considering a penalized problem in $\mathcal{H}_{2} \subset L^{2}((0, T) \times \Omega)^{m}$ : the behavior at time $t=T$ of the weight $\rho_{0}$ will be the key point to produce more regular controls in $L^{p}$-sense.

\subsubsection{The beginning of the Penalized Hilbert Uniqueness Method}

Let us fix $Z_{0} \in L_{\text {inv }}^{2}$.

We define $P_{\varepsilon}: \mathcal{H}_{2} \rightarrow \mathbb{R}^{+}$, by, for every $H^{J} \in \mathcal{H}_{2}$,

$$
P_{\varepsilon}\left(H^{J}\right):=\frac{1}{2} \iint_{(0, T) \times \omega} \rho_{0}^{-2}(t)\left|H^{J}(t, x)\right|^{2} \mathrm{~d} x \mathrm{~d} t+\frac{1}{2 \varepsilon}\|Z(T, .)\|_{L^{2}(\Omega)^{n}}^{2},
$$

where $Z$ is the solution to the Cauchy problem (L-Z) (see Sect. 2.2) associated to the control $H^{J}$.

The functional $P_{\varepsilon}$ is a $C^{1}$, coercive, strictly convex functional on the Hilbert space $\mathcal{H}_{2}$, then $P_{\varepsilon}$ has a unique minimum $H^{J, \varepsilon} \in \mathcal{H}_{2}$. Let $Z^{\varepsilon}$ be the solution to the Cauchy problem (L-Z) with control $H^{J, \varepsilon}$ and initial data $Z_{0}$.

The Euler-Lagrange equation gives

$$
\forall H^{J} \in \mathcal{H}_{2}, \quad \iint_{(0, T) \times \omega} \rho_{0}^{-2} H^{J, \varepsilon} \cdot H^{J} \mathrm{~d} x \mathrm{~d} t+\frac{1}{\varepsilon} \int_{\Omega} Z^{\varepsilon}(T, x) \cdot Z(T, x) \mathrm{d} x=0,
$$

where $Z$ is the solution to the Cauchy problem (L-Z) associated to the control $H^{J}$ and initial data $Z_{0}=0$.

We introduce $\varphi^{\varepsilon}$ the solution to the adjoint problem (4.8) with final condition $\varphi^{\varepsilon}(T,)=.-\frac{1}{\varepsilon} Z^{\varepsilon}(T,$.$) . A$ duality argument between $Z$ and $\varphi^{\varepsilon}$ gives

$$
-\frac{1}{\varepsilon} \int_{\Omega} Z(T, x) \cdot Z^{\varepsilon}(T, x) \mathrm{d} x=\int_{\Omega} Z(T, x) \cdot \varphi^{\varepsilon}(T, x) \mathrm{d} x=\iint_{(0, T) \times \omega} H^{J} \cdot \varphi^{\varepsilon} .
$$


Then, we deduce from (5.3) and (5.4) that

$$
\forall H^{J} \in \mathcal{H}_{2}, \iint_{(0, T) \times \omega} \rho_{0}^{-2} H^{J, \varepsilon} \cdot H^{J}=\iint_{(0, T) \times \omega} \varphi^{\varepsilon} \cdot H^{J} .
$$

Consequently, we have

$$
\forall i \in\{1, \ldots, m\}, h_{i}^{\varepsilon}=\rho_{0}^{2} \varphi_{i}^{\varepsilon} 1_{\omega}
$$

Another duality argument applied between $Z^{\varepsilon}$ and $\varphi^{\varepsilon}$ together with (5.5) gives

$$
\begin{aligned}
-\frac{1}{\varepsilon} \int_{\Omega}\left|Z^{\varepsilon}(T, x)\right|^{2} \mathrm{~d} x & =\int_{\Omega} Z^{\varepsilon}(T, x) \cdot \varphi^{\varepsilon}(T, x) \mathrm{d} x \\
& =\int_{\Omega} Z_{0}(x) \cdot \varphi^{\varepsilon}(0, x) \mathrm{d} x+\iint_{(0, T) \times \omega} H^{J, \varepsilon} \cdot \varphi^{\varepsilon}
\end{aligned}
$$

which yields

$$
-\frac{1}{\varepsilon}\left\|Z^{\varepsilon}(T, .)\right\|_{L^{2}(\Omega)^{n}}^{2}=\int_{\Omega} Z_{0}(x) \cdot \varphi^{\varepsilon}(0, x) \mathrm{d} x+\sum_{i=1}^{m} \iint_{(0, T) \times \omega}\left|\rho_{0} \varphi_{i}^{\varepsilon}\right|^{2} .
$$

By Young's inequality and the observability estimate (4.9) applied to $\varphi^{\varepsilon}$, for $\delta>0$, we have:

$$
\begin{aligned}
& \left|\int_{\Omega} Z_{0}(x) \cdot \varphi^{\varepsilon}(0, x) \mathrm{d} x\right| \\
& \leq \delta\left\|\varphi^{\varepsilon}(0, .)\right\|_{L^{2}(\Omega)^{n}}^{2}+C_{\delta}\left\|Z_{0}\right\|_{L^{2}(\Omega)^{n}}^{2} \\
& \leq \delta C_{T}\left(\sum_{i=1}^{m} \iint_{(0, T) \times \omega}\left|\rho_{0}(t) \varphi_{i}^{\varepsilon}(t, x)\right|^{2} \mathrm{~d} x \mathrm{~d} t\right)+C_{\delta}\left\|Z_{0}\right\|_{L^{2}(\Omega)^{n}}^{2} .
\end{aligned}
$$

Then, by using (5.5), (5.6), (5.7) and by taking $\delta$ sufficiently small, we get

$$
\frac{1}{\varepsilon}\left\|Z^{\varepsilon}(T, .)\right\|_{L^{2}(\Omega)^{n}}^{2}+\frac{1}{2}\left\|\rho_{0}^{-1} H^{J, \varepsilon}\right\|_{L^{2}((0, T) \times \omega)^{n}}^{2} \leq C_{T}\left\|Z_{0}\right\|_{L^{2}(\Omega)^{n}}^{2} .
$$

Remark 5.2. The estimate (5.8) yields Proposition 4.3 for $S=0$ by letting $\varepsilon \rightarrow 0$. We remark that we have only used the term $\|\varphi(0, .)\|_{L^{2}(\Omega)^{n}}^{2}$ in the left hand side of (4.9). The second term in the left hand side of (4.9) enables to get more regularity (in $L^{p}$-sense) for the control $H^{J}$ (see Sect. 5.1.2).

\subsubsection{Bootstrap method}

In the next two parts, we will use the key identity between the control $H^{J, \varepsilon}$ and the solution of the adjoint system $\varphi^{\varepsilon}$, i.e., (5.5) in order to deduce $L^{p}$-regularity for $H^{J, \varepsilon}$ from $L^{p}$-regularity for $\varphi^{\varepsilon}$. This kind of regularity will come from the application of successive $L^{p}$-parabolic regularity theorems stated in Proposition A.4 to a modification of $\varphi^{\varepsilon}$ called $\psi^{\varepsilon, r}$ (see a precise definition in (5.13) below) which is bounded from below by $\rho_{0}^{2} \varphi$. The beginning of this bootstrap argument is the strong observability inequality (4.9). Finally, we will pass to the limit $(\varepsilon \rightarrow 0)$ in $\frac{1}{\varepsilon}\left\|Z^{\varepsilon}(T, .)\right\|_{L^{2}(\Omega)^{n}}^{2} \leq C_{T}\left\|Z_{0}\right\|_{L^{2}(\Omega)^{n}}^{2}$ coming from (5.8) and $\left\|H^{J, \varepsilon}\right\|_{L^{\infty}\left(Q_{T}\right)} \leq C_{T}\left\|Z_{0}\right\|_{L^{2}(\Omega)^{n}}$ coming from (5.22) (see below). 
By using Remark 4.1, we introduce the positive real number

$$
\gamma:=2 p-(1+p) q^{2}>0 .
$$

Let us define a sequence of increasing positive real numbers $\left(\gamma_{r}\right)_{r \in \mathbb{N}}$ such that $\lim _{r \rightarrow+\infty} \gamma_{r}=\gamma$, where $\gamma$ is defined in (5.9).

We introduce for every $r \in \mathbb{N}$,

$$
\rho_{\mathcal{S}, r}(t):=M^{-1-p} \exp \left(-\frac{\left((1+p) q^{2}+\gamma_{r}\right) M}{(q-1)(T-t)}\right) .
$$

Then, we have from (4.1), for every $r \in \mathbb{N}$,

$$
\rho_{0}^{2} \leq C_{T} \rho_{\mathcal{S}, r}
$$

We remark that we have for every $r \in \mathbb{N}$,

$$
\left|\rho_{\mathcal{S}, r+1}^{\prime}(t)\right| \leq C_{T, r} \rho_{\mathcal{S}, r}(t) .
$$

We define for every $r \in \mathbb{N}$,

$$
\psi^{\varepsilon, r}(t, x):=\rho_{\mathcal{S}, r}(t) \varphi^{\varepsilon}(t, x) .
$$

From (4.8), (5.10) and (5.13), we have for every $r \in \mathbb{N}^{*}$,

$$
\begin{cases}-\partial_{t} \psi^{\varepsilon, r}-D_{J}^{\operatorname{tr}} \Delta \psi^{\varepsilon, r}=A_{J}^{\operatorname{tr}} \psi^{\varepsilon, r}-\rho_{\mathcal{S}, r}^{\prime}(t) \varphi^{\varepsilon} & \text { in }(0, T) \times \Omega, \\ \frac{\partial \psi^{\varepsilon, r}}{\partial \nu}=0 & \text { on }(0, T) \times \partial \Omega, \\ \psi^{\varepsilon, r}(T, .)=0 & \text { in } \Omega .\end{cases}
$$

By using (5.12), we remark that

$$
\left|-\rho_{\mathcal{S}, r}^{\prime}(t) \varphi^{\varepsilon}\right| \leq C_{T}\left|\psi^{\varepsilon, r-1}\right| .
$$

Let $\left(p_{r}\right)_{r \in \mathbb{N}}$ be the following sequence defined by induction

$$
\begin{gathered}
p_{0}=2, \\
p_{r+1}:=\left\{\begin{array}{cl}
\frac{(N+2) p_{r}}{N+2-2 p_{r}} & \text { if } p_{r}<\frac{N+2}{2}, \\
2 p_{r} & \text { if } p_{r}=\frac{N+2}{2}, \\
+\infty & \text { if } p_{r}>\frac{N+2}{2} .
\end{array}\right.
\end{gathered}
$$

There exists $l \in \mathbb{N}^{*}$ such that

$$
\forall r \geq l, p_{r}=+\infty
$$

We show, by induction, that for every $0 \leq r \leq l$, we have

$$
\psi^{\varepsilon, r} \in L^{p_{r}}\left(Q_{T}\right)^{n} \text { and }\left\|\psi^{\varepsilon, r}\right\|_{L^{p_{r}}\left(Q_{T}\right)^{n}} \leq C_{T}\left\|Z_{0}\right\|_{L^{2}(\Omega)^{n}} .
$$


The case $r=0$ can be deduced from the fact that $\gamma_{0}>0$ and the observability estimate (4.9) $\left(p_{0}=2\right.$ by (5.16)).

Let $r \in \mathbb{N}^{*}$. We assume that

$$
\psi^{\varepsilon, r-1} \in L^{p_{r-1}}\left(Q_{T}\right)^{n} \text { and }\left\|\psi^{\varepsilon, r-1}\right\|_{L^{p_{r-1}}\left(Q_{T}\right)^{n}} \leq C_{T}\left\|Z_{0}\right\|_{L^{2}(\Omega)^{n}} .
$$

Then, from (5.14), (5.15), (5.20) and from the maximal regularity theorem: Proposition A.4 applied with $p_{r-1} \in$ $(1,+\infty)$, we get

$$
\psi^{\varepsilon, r} \in X_{p_{r-1}}^{n} \text { and }\left\|\psi^{\varepsilon, r}\right\|_{X_{p_{r-1}}^{r}} \leq C_{T}\left\|Z_{0}\right\|_{L^{2}(\Omega)^{n}} .
$$

Moreover, by the Sobolev embedding: Proposition A.5 and (5.17), we have

$$
\psi^{\varepsilon, r} \in L^{p_{r}}\left(Q_{T}\right)^{n} \text { and }\left\|\psi^{\varepsilon, r}\right\|_{L^{p_{r}}\left(Q_{T}\right)^{n}} \leq C_{T}\left\|Z_{0}\right\|_{L^{2}(\Omega)^{n}} .
$$

This concludes the induction.

\subsubsection{The end of the Penalized Hilbert Uniqueness Method}

Now, by applying consecutively (5.18) $\left(p_{l}=+\infty\right),(5.5),(5.11)$ and $(5.19)$, we have for every $i \in\{1, \ldots, m\}$,

$$
\left\|h_{i}^{\varepsilon}\right\|_{L^{\infty}\left(Q_{T}\right)} \leq C_{T}\left\|Z_{0}\right\|_{L^{2}(\Omega)^{n}} .
$$

Therefore, from $(5.22),\left(H^{J, \varepsilon}\right)_{\varepsilon}$ is uniformly bounded in $L^{\infty}\left(Q_{T}\right)^{m}$, then up to a subsequence, we can assume that there exists $H^{J} \in L^{\infty}\left(Q_{T}\right)^{m}$ such that

$$
\begin{gathered}
H^{J, \varepsilon} \underset{\varepsilon \rightarrow 0}{\rightarrow}{ }^{*} H^{J} \text { in } L^{\infty}\left(Q_{T}\right)^{m}, \\
\left\|H^{J}\right\|_{L^{\infty}\left(Q_{T}\right)^{m}} \leq C_{T}\left\|Z_{0}\right\|_{L^{2}(\Omega)^{n}} .
\end{gathered}
$$

From (5.22), Proposition A.2 applied to (L-Z) satisfied by $Z^{\varepsilon}$, we obtain

$$
\left\|Z^{\varepsilon}\right\|_{W_{T}^{n}} \leq C_{T}\left\|Z_{0}\right\|_{L^{2}(\Omega)^{n}} .
$$

So, from (5.25), up to a subsequence, we can suppose that there exists $Z \in W_{T}^{n}$ such that

$$
Z^{\varepsilon} \underset{\varepsilon \rightarrow 0}{\rightarrow} Z \text { in } L^{2}\left(0, T ; H^{1}(\Omega)^{n}\right), \partial_{t} Z^{\varepsilon} \underset{\varepsilon \rightarrow 0}{\rightarrow} \partial_{t} Z \text { in } L^{2}\left(0, T ;\left(H^{1}(\Omega)\right)^{\prime n}\right),
$$

and from (1.11),

$$
Z^{\varepsilon}(0, .) \underset{\varepsilon \rightarrow 0}{\rightarrow} Z(0, .) \text { in } L^{2}(\Omega)^{n}, Z^{\varepsilon}(T, .) \underset{\varepsilon \rightarrow 0}{\rightarrow} Z(T, .) \text { in } L^{2}(\Omega)^{n} .
$$

Then, as we have $Z^{\varepsilon}(0,)=.Z_{0}, Z^{\varepsilon}(T,.) \rightarrow 0$ from (5.8) and by uniqueness of the limit, we deduce that

$$
Z(0, .)=Z_{0} \text {, and } Z(T, .)=0 .
$$

By letting $\varepsilon \rightarrow 0$, we have from (5.26), (5.23) and (5.28) that $Z$ is a solution to (L-Z) satisfying $Z(T,)=$. which conludes the proof of Theorem 5.1 by using (5.24). 


\subsection{The come back to the source term method in $L^{\infty}$}

The goal of this section is to apply the source term method in $L^{\infty}$ thanks to the null-controllability result in $L^{\infty}$ : Theorem 5.1 .

To simplify the notations, we assume that the control cost in $L^{\infty}$ of Theorem 5.1 satisfies: $C_{T} \leq M e^{M / T}$ where $M$ is already defined at the beginning of Section 4.

From Proposition A.11 with $r=+\infty$ proved in Appendix A.6, we deduce the following null-controllability result for $(\mathrm{L}+\mathrm{S}-\mathrm{Z})$ (see Sect. 4$)$ in $L^{\infty}$.

Proposition 5.3. For every $S \in \mathcal{S}_{\infty}$ and $Z_{0} \in L_{\mathrm{inv}}^{\infty}$, there exists $H^{J} \in \mathcal{H}_{\infty}$, such that the solution $Z$ of $(\mathrm{L}+\mathrm{S}-\mathrm{Z})$ satisfies $Z \in \mathcal{Z}_{\infty}$. Furthermore, there exists $C>0$, not depending on $S$ and $Z_{0}$, such that

$$
\left\|Z / \rho_{0}\right\|_{L^{\infty}\left([0, T] ; L^{\infty}(\Omega)^{n}\right)}+\left\|H^{J}\right\|_{\mathcal{H}_{\infty}} \leq C\left(\left\|Z_{0}\right\|_{L^{\infty}(\Omega)^{n}}+\|S\|_{\mathcal{S}_{\infty}}\right)
$$

In particular, since $\rho_{0}$ is a continuous function satisfying $\rho_{0}(T)=0$, the above relation $(5.29)$ yields $Z(T,)=$.0 .

\section{The INVERSE MAPPING THEOREM IN APPROPRIATE SPACES}

The goal of this section is to prove Theorem 2.3. The proof is based on Proposition 5.3 and an inverse mapping theorem in suitable spaces. It uses similar arguments to those employed, for instance, in [16].

Proof. Let us introduce the following space (see the Defs. (4.4), (4.5) and (4.6)):

$$
E:=\left\{\left(Z, H^{J}\right) \in \mathcal{Z}_{\infty} \times \mathcal{H}_{\infty} ; \partial_{t} Z-D_{J} \Delta Z-A_{J} Z-H^{J} 1_{\omega} \in S_{\infty}\right\}
$$

We endow $E$ with the following norm: for every $\left(Z, H^{J}\right) \in E$,

$$
\left\|\left(Z, H^{J}\right)\right\|_{E}=\|Z(0, .)\|_{L^{\infty}}+\|Z\|_{\mathcal{Z}_{\infty}}+\left\|H^{J}\right\|_{\mathcal{H}_{\infty}}+\left\|\partial_{t} Z-D_{J} \Delta Z-A_{J} Z-H^{J} 1_{\omega}\right\|_{S_{\infty}} .
$$

Then, $\left(E,\|\cdot\|_{E}\right)$ is a Banach space.

For every $Z \in \mathcal{Z}_{\infty}$, we introduce the following polynomial nonlinearity of degree more than 2 :

$$
Q(Z):=G(Z)-A_{J} Z
$$

where $G$ is defined in (2.3). By denoting $\gamma:=\max \left(\sum_{i=1}^{n} \alpha_{i}, \sum_{i=1}^{n} \beta_{i}\right)$, we remark that for every $Z \in \mathcal{Z}_{\infty}, Q(Z)=$ $\sum_{i=2}^{\gamma} Q_{i}(Z)$ where for every $2 \leq i \leq \gamma, Q_{i}(Z)$ is a polynomial term with respect to $Z=\left(z_{1}, \ldots, z_{n}\right)$ of degree $i$. By using (4.3), we deduce that $Q(Z) \in \mathcal{S}_{\infty}$ and for every $2 \leq i \leq \gamma$,

$$
\left\|Q_{i}(Z)\right\|_{\mathcal{S}_{\infty}}=\left\|\frac{Q_{i}(Z)}{\rho_{\mathcal{S}}}\right\|_{L^{\infty}\left(Q_{T}\right)^{n}}=\left\|\rho_{0}^{i-2} \frac{\rho_{0}^{2}}{\rho_{\mathcal{S}}} \frac{Q_{i}(Z)}{\rho_{0}^{i}}\right\|_{L^{\infty}\left(Q_{T}\right)^{n}} \leq C\|Z\|_{\mathcal{Z}_{\infty}}^{i}
$$

We introduce the following mapping:

$$
\begin{aligned}
& \mathcal{A}: \quad E \quad \longrightarrow F:=\mathcal{S}_{\infty} \times L_{\mathrm{inv}}^{\infty} \\
& (Z, H) \longmapsto\left(\partial_{t} Z-D_{J} \Delta Z-A_{J} Z-H^{J} 1_{\omega}-Q(Z), Z(0, .)\right) \text {. }
\end{aligned}
$$

By using (6.1), the fact that for $\left(Z, H^{J}\right) \in E$ and $Q(Z) \in \mathcal{S}_{\infty}$ by (6.4), we see that $\mathcal{A}$ is well-defined. Moreover, $\mathcal{A} \in C^{1}(E ; F)$. Indeed, all the terms in (6.5) are linear and continuous (thus $C^{\infty}$ ) thanks to (6.2) except the 
term $Q(Z)$. And, for $\left(Z, H^{J}\right) \in E, Q(Z)$ is a polynomial function with respect to $Z$ which is $C^{\infty}$ thanks to (6.4).

Moreover, the differential of $\mathcal{A}$ at the point $(0,0)$ in the direction $\left(Z, H^{J}\right)$ is

$$
D \mathcal{A}(0,0) \cdot\left(Z, H^{J}\right)=\left(\partial_{t} Z-D_{J} \Delta Z-A_{J} Z-H^{J} 1_{\omega}, Z(0, .)\right)
$$

which is onto by using Proposition 5.3. Then, by using the inverse mapping theorem (see [9], Thm. 2), we deduce that there exists $r>0$, such that for every $\left(S, Z_{0}\right) \in F$ satisfying $\left\|\left(S, Z_{0}\right)\right\|_{F} \leq r$, there exists $\left(Z, H^{J}\right) \in E$ such that $\mathcal{A}\left(Z, H^{J}\right)=\left(S, Z_{0}\right)$. By taking $S=0$ and $Z_{0} \in L_{\mathrm{inv}}^{\infty}$ such that $\left\|Z_{0}\right\|_{L^{\infty}(\Omega)^{n}} \leq r$, we get the existence of $\left(Z, H^{J}\right) \in \mathcal{Z}_{\infty} \times \mathcal{H}_{\infty}$ such that

$$
\begin{cases}\partial_{t} Z-D_{J} \Delta Z=\underbrace{A_{J} Z+Q(Z)}_{G(Z) \text { by }(6.3)}+H^{J} 1_{\omega} & \text { in }(0, T) \times \Omega \\ \frac{\partial Z}{\partial \nu}=0 & \text { on }(0, T) \times \partial \Omega \\ (Z(0, .), Z(T, .))=\left(Z_{0}, 0\right) & \text { in } \Omega\end{cases}
$$

This concludes the proof of Theorem 2.3.

\section{Comments}

\subsection{More general semilinearities}

In this paper, we have only considered particular semilinearities of the form (1.6). But the main result of the article, i.e., Theorem 1.7 holds true with more general polynomial semilinearities satisfying

$$
\exists R \in \mathbb{R}\left[X_{1}, \ldots, X_{n}\right], \forall 1 \leq i \leq n, \exists a_{i} \in \mathbb{R}^{*}, f_{i}=a_{i} R
$$

where $\mathbb{R}\left[X_{1}, \ldots, X_{n}\right]$ denotes the space of multivariate polynomials with coefficients in $\mathbb{R}$. In this case, $\left(u_{i}^{*}\right)_{1 \leq i \leq n}$ is a constant nonnegative stationary state if

$$
\left(u_{i}^{*}\right)_{1 \leq i \leq n} \in[0,+\infty)^{n} \text { and } R\left(u_{1}^{*}, \ldots, u_{n}^{*}\right)=0 .
$$

\subsection{Degenerate cases}

In this part, we assume that Assumption 1.6 is not satisfied. Then, the usual strategy is to perform the return method, introduced by Jean-Michel Coron in [7] (see also [8], Chap. 6). This method consists in finding a reference trajectory $\left(\bar{U}, \overline{H^{J}}\right.$ ) verifying $U(0,)=.U(T,)=.U^{*}$ of (NL-U) (see Sect. 1.2) such that the linearized system of (NL-U) around $\left(\bar{U}, \overline{H^{J}}\right)$ is null-controllable (see $[10]$ for the first application of this method in the context of the null-controllability of reaction-diffusion systems).

Example 7.1. We come back to Application 1.11 with $J=\{1-3\}$. In this case, (1.20) is not satisfied if and only if $\left(u_{1}^{*}, u_{3}^{*}, u_{4}^{*}\right)=(0,0,0)$. More precisely, the linearized system around $\left(\left(0, u_{2}^{*}, 0,0\right),(0,0,0)\right)$ is not null-controllable because the fourth equation is decoupled from the others:

$$
\partial_{t} u_{4}-d_{4} \Delta u_{4}=-u_{2}^{*} u_{4}
$$

By using the return method, the author proves the local null-controllability around $\left(0, u_{2}^{*}, 0,0\right)$ of (NL-U) (see [24], Sect. 4.1.1.2). 


\section{Appendix A.}

\section{A.1 Toolbox for linear parabolic systems}

\section{A.1.1 Well-posedness results}

Definition A.1. Let $k \in \mathbb{N}^{*}, D \in \mathcal{M}_{k}(\mathbb{R})$ a diagonalizable matrix such that $\operatorname{Sp}(D) \subset(0,+\infty), A \in \mathcal{M}_{k}(\mathbb{R})$, $U_{0} \in L^{2}(\Omega)^{k}, S \in L^{2}\left(Q_{T}\right)^{k}$. A function $U \in W_{T}^{k}$ is a solution to

$$
\begin{cases}\partial_{t} U-D \Delta U=A U+S & \text { in }(0, T) \times \Omega \\ \frac{\partial U}{\partial \nu}=0 & \text { on }(0, T) \times \partial \Omega \\ U(0, .)=U_{0} & \text { in } \Omega\end{cases}
$$

if for every $V \in L^{2}\left(0, T ; H^{1}(\Omega)^{k}\right.$,

$$
\int_{0}^{T}\left(\partial_{t} U, V\right)_{\left.\left(H^{1}(\Omega)^{k}\right)^{\prime}, H^{1}(\Omega)^{k}\right)}+\int_{Q_{T}} D \nabla U . \nabla V=\int_{Q_{T}}(A U+S) \cdot V,
$$

and

$$
U(0, .)=U_{0} \text { in } L^{2}(\Omega)^{k}
$$

The following well-posedness result in $L^{2}$ holds for linear parabolic equations.

Proposition A.2. With the same notations as in Definition A.1, the Cauchy problem (A.1) admits a unique solution $U \in W_{T}^{k}$. Moreover, there exists $C>0$ independent of $U_{0}$ and $S$ such that

$$
\|U\|_{W_{T}^{k}} \leq C\left(\left\|U_{0}\right\|_{L^{2}(\Omega)^{k}}+\|S\|_{L^{2}\left(Q_{T}\right)^{k}}\right) .
$$

We also have the following $L^{\infty}$-estimate for (A.1).

Proposition A.3. With the same notations as in Definition A.1, the unique solution $U$ of (A.1) satisfies

$$
\|U\|_{L^{\infty}\left(Q_{T}\right)^{k}} \leq C\left(\left\|U_{0}\right\|_{L^{\infty}(\Omega)^{k}}+\|S\|_{L^{\infty}\left(Q_{T}\right)^{k}}\right) .
$$

with a constant $C>0$ independent of $U_{0}$ and $S$.

The proofs of Propositions A.2 and A.3 can be found in ([24], Prop. 2.3).

\section{A.1.2 Maximal regularity theorems and Sobolev embeddings}

In this part, we recall a maximal regularity theorem in $L^{p}(1<p<+\infty)$ for parabolic systems and an embedding result for Sobolev spaces.

We introduce the following spaces: for every $r \in[1,+\infty]$,

$$
\begin{gathered}
W_{N e}^{2, r}(\Omega):=\left\{u \in W^{2, r}(\Omega) ; \frac{\partial u}{\partial \nu}=0\right\}, \\
X_{r}:=L^{r}\left(0, T ; W_{N e}^{2, r}(\Omega)\right) \cap W^{1, r}\left(0, T ; L^{r}(\Omega)\right) .
\end{gathered}
$$

We have the following maximal regularity theorem. 
Proposition A.4. ([11], Thm. 2.1)

Let $1<r<+\infty, k \in \mathbb{N}^{*}, D \in \mathcal{M}_{k}(\mathbb{R})$ such that $\operatorname{Sp}(D) \subset(0,+\infty), A \in \mathcal{M}_{k}(\mathbb{R})$ and $S \in L^{r}\left(Q_{T}\right)^{k}$. The solution $U$ of (A.1) satisfies

$$
\|U\|_{X_{r}^{k}} \leq C\|S\|_{L^{r}\left(Q_{T}\right)^{k}}
$$

with $C$ independent of $S$.

We have the following embedding result for Sobolev spaces.

Proposition A.5. ([34], Thm. 1.4.1)

Let $r \in[1,+\infty[$, we have

$$
X_{r} \hookrightarrow\left\{\begin{array}{cc}
L^{\frac{(N+2) r}{N+2-2 r}}\left(Q_{T}\right) & \text { if } r<\frac{N+2}{2} \\
L^{2 r}\left(Q_{T}\right) & \text { if } r=\frac{N+2}{N^{2}} \\
L^{\infty}\left(Q_{T}\right) & \text { if } r>\frac{N+2}{2} .
\end{array}\right.
$$

\section{A.2 Stationary states}

We only have considered nonnegative stationary constant solutions of (1.3). It is not restrictive because of the following proposition.

Proposition A.6. Let $\left(u_{i}\right)_{1 \leq i \leq n} \in C^{2}(\bar{\Omega})^{n}$ be a nonnegative solution of

$$
\forall 1 \leq i \leq n, \begin{cases}-d_{i} \Delta u_{i}=f_{i}(U) & \text { in } \Omega \\ \frac{\partial u_{i}}{\partial \nu}=0 & \text { on } \partial \Omega\end{cases}
$$

where $f_{i}(U)(1 \leq i \leq n)$ is defined in (1.6). Then, for every $1 \leq i \leq n, u_{i}$ is constant.

The proof relies on an entropy inequality: $\sum_{i=1}^{n} \log \left(u_{i}\right) f_{i}(U) \leq 0$.

Proof. Let $\varepsilon>0$ be a small parameter. For every $1 \leq i \leq n$, we introduce

$$
u_{i, \varepsilon}=u_{i}+\varepsilon, \quad w_{i, \varepsilon}=u_{i, \varepsilon}\left(\log u_{i, \varepsilon}-1\right)+1 \geq 0 .
$$

We have

$$
\forall 1 \leq i \leq n, \nabla w_{i, \varepsilon}=\log \left(u_{i, \varepsilon}\right) \nabla u_{i, \varepsilon}, \quad \Delta w_{i, \varepsilon}=\log \left(u_{i, \varepsilon}\right) \Delta u_{i, \varepsilon}+\frac{\left|\nabla u_{i, \varepsilon}\right|^{2}}{u_{i, \varepsilon}}
$$

Then, from (A.5) and (A.6), we have

$$
\forall 1 \leq i \leq n, \begin{cases}-d_{i} \Delta w_{i, \varepsilon}+d_{i} \frac{\left|\nabla u_{i, \varepsilon}\right|^{2}}{u_{i, \varepsilon}}=\log \left(u_{i, \varepsilon}\right) f_{i}(U) & \text { in } \Omega, \\ \frac{\partial w_{i, \varepsilon}}{\partial n}=0 & \text { on } \partial \Omega .\end{cases}
$$

We sum the $n$ equations of (A.7), we integrate on $\Omega$ and we use the increasing of the function log:

$$
\begin{aligned}
& \int_{\Omega} \sum_{i=1}^{n} d_{i} \frac{\left|\nabla u_{i, \varepsilon}\right|^{2}}{u_{i, \varepsilon}} \\
& =-\left(\int_{\Omega}\left\{\log \left(\prod_{i=1}^{n} u_{i, \varepsilon}^{\alpha_{i}}\right)-\log \left(\prod_{i=1}^{n} u_{i, \varepsilon}^{\beta_{i}}\right)\right\}\left\{\prod_{i=1}^{n} u_{i}^{\alpha_{i}}-\prod_{i=1}^{n} u_{i}^{\beta_{i}}\right\}\right)
\end{aligned}
$$




$$
\begin{aligned}
& =-\left(\int_{\Omega}\left\{\log \left(\prod_{i=1}^{n} u_{i, \varepsilon}^{\alpha_{i}}\right)-\log \left(\prod_{i=1}^{n} u_{i, \varepsilon}^{\beta_{i}}\right)\right\}\left\{\prod_{i=1}^{n} u_{i, \varepsilon}^{\alpha_{i}}-\prod_{i=1}^{n} u_{i, \varepsilon}^{\beta_{i}}+\mathcal{O}(\varepsilon)\right\}\right) \\
& \leq \int_{\Omega}\left|\log \left(\prod_{i=1}^{n} u_{i, \varepsilon}^{\alpha_{i}}\right)-\log \left(\prod_{i=1}^{n} u_{i, \varepsilon}^{\beta_{i}}\right)\right| \mathcal{O}(\varepsilon) \leq\left(\sum_{i=1}^{n}\left(\alpha_{i}+\beta_{i}\right)\right)|\log (\varepsilon)| \mathcal{O}(\varepsilon) \underset{\varepsilon \rightarrow 0}{\rightarrow} 0 .
\end{aligned}
$$

Moreover,

$$
\forall 1 \leq i \leq n, \int_{\Omega} d_{i} \frac{\left|\nabla u_{i}^{\varepsilon}\right|^{2}}{u_{i}^{\varepsilon}}=\int_{\Omega} 4 d_{i}\left|\nabla \sqrt{u_{i}^{\varepsilon}}\right|^{2}
$$

Consequently, from (A.8), (A.9), we get that

$$
\forall 1 \leq i \leq n, \int_{\Omega} 4 d_{i}\left|\nabla \sqrt{u_{i}}\right|^{2}=0
$$

Therefore, for every $1 \leq i \leq n, u_{i}$ is constant.

Our proof of Theorem 1.7 does not treat the case of stationary states which can change of sign, contrary to the proof of ([24], Thm. 3.2) (see [24], Sect. 6.2).

\section{A.3 Proof of the existence of invariant quantities in the system}

The goal of this section is to prove Proposition 1.3.

Proof. We introduce the notation $R:=\prod_{k=1}^{n} u_{k}^{\alpha_{k}}-\prod_{k=1}^{n} u_{k}^{\beta_{k}}$.

Let $i \in\{m+1, \ldots, n\}$. By using the fact that $u_{i} \in W_{T}$ and from ([12], Lem. 3), we obtain that the mapping $t \mapsto \int_{\Omega} u_{i}(t, x) \mathrm{d} x$ is absolutely continuous and for a.e. $0 \leq t \leq T$,

$$
\frac{\mathrm{d}}{\mathrm{d} t} \int_{\Omega} u_{i}(t, x) \mathrm{d} x=\left(\partial_{t} u_{i}(t, .), 1\right)_{\left(H^{1}(\Omega)\right)^{\prime}, H^{1}(\Omega)} .
$$

Then, by using that $\left(\left(u_{i}\right)_{1 \leq i \leq n},\left(h_{i}\right)_{1 \leq i \leq m}\right)$ is a trajectory of $(\mathrm{NL}-\mathrm{U})$ and by taking $w=(0, \ldots, 0, \underbrace{1}_{i}, 0, \ldots, 0)^{\operatorname{tr}}$ in (1.12), we find that for a.e. $0 \leq t \leq T$,

$$
\begin{aligned}
\left(\partial_{t} u_{i}(t, .), w\right)_{\left(H^{1}(\Omega)\right)^{\prime}, H^{1}(\Omega)} & =d_{i}\left(\nabla u_{i}(t, .), \nabla w\right)_{L^{2}(\Omega), L^{2}(\Omega)}+\int_{\Omega}\left(\beta_{i}-\alpha_{i}\right) R \\
& =\int_{\Omega}\left(\beta_{i}-\alpha_{i}\right) R .
\end{aligned}
$$

Then, by using (A.10) and (A.11), we get for a.e. $0 \leq t \leq T$,

$$
\frac{\mathrm{d}}{\mathrm{d} t} \int_{\Omega} \frac{u_{i}(t, .)}{\beta_{i}-\alpha_{i}}=\int_{\Omega} R
$$

Now, let $m+1 \leq k \neq l \leq n$. By (A.12) for $i=k$ and (A.12) for $i=l$, we deduce that for a.e. $0 \leq t \leq T$,

$$
\frac{\mathrm{d}}{\mathrm{d} t} \int_{\Omega}\left(\frac{u_{k}(t, .)}{\beta_{k}-\alpha_{k}}-\frac{u_{l}(t, .)}{\beta_{l}-\alpha_{l}}\right)=0 .
$$


Therefore, from (A.13), we have for every $t \in[0, T]$,

$$
\frac{1}{|\Omega|} \int_{\Omega}\left(\frac{u_{k}(t, x)}{\beta_{k}-\alpha_{k}}-\frac{u_{l}(t, x)}{\beta_{l}-\alpha_{l}}\right) \mathrm{d} x=\frac{u_{k}^{*}}{\beta_{k}-\alpha_{k}}-\frac{u_{l}^{*}}{\beta_{l}-\alpha_{l}}
$$

If we assume that $d:=d_{k}=d_{l}$, then the equation satisfied by $v:=\left(\beta_{l}-\alpha_{l}\right) u_{k}-\left(\beta_{k}-\alpha_{k}\right) u_{l}$ is

$$
\begin{cases}\partial_{t} v-\mathrm{d} \Delta v=0 & \text { in }(0, T) \times \Omega \\ \frac{\partial v}{\partial \nu}=0 & \text { on }(0, T) \times \partial \Omega \\ v(T, .)=\left(\beta_{l}-\alpha_{l}\right) u_{k}^{*}-\left(\beta_{k}-\alpha_{k}\right) u_{l}^{*} & \text { in } \Omega\end{cases}
$$

The backward uniqueness of the heat equation (see for instance [4], Thm. II.1) applied to (A.14) leads to

$$
\forall t \in[0, T],\left(\beta_{l}-\alpha_{l}\right) u_{k}(t, .)-\left(\beta_{k}-\alpha_{k}\right) u_{l}(t, .)=\left(\beta_{l}-\alpha_{l}\right) u_{k}^{*}-\left(\beta_{k}-\alpha_{k}\right) u_{l}^{*} .
$$

This yields (1.15).

\section{A.4 Proofs concerning the change of variables}

\section{A.4.1 Proof of the equivalence of the two systems}

In this section, we prove Proposition 2.1. It is based on the following algebraic lemma.

Lemma A.7. Let $s$ be an integer such that $s \geq 2$. Let $\left(a_{1}, \ldots, a_{s}\right) \in \mathbb{C}^{s}$ be such that $a_{i} \neq a_{j}$ for $i \neq j$. Then, we have

$$
\sum_{i=1}^{s} \prod_{\substack{j=1 \\ j \neq i}}^{s} \frac{1}{a_{i}-a_{j}}=0
$$

Proof. Let $\mathbb{C}(X)$ be the field of fractional functions with coefficients in $\mathbb{C}$ and $F \in \mathbb{C}(X)$ be defined by

$$
F(X):=\left(\sum_{i=1}^{s-1}\left(\prod_{\substack{j=1 \\ j \neq i}}^{s-1} \frac{1}{a_{i}-a_{j}}\right) \frac{1}{a_{i}-X}\right)+\prod_{j=1}^{s-1} \frac{1}{X-a_{j}} .
$$

The partial fractional decomposition of $F$ is the following one:

$$
F(X)=\sum_{i=1}^{s-1} \frac{b_{i}}{X-a_{i}}, \quad \text { where } b_{i} \in \mathbb{C} .
$$

For $1 \leq i \leq s-1$, we compute each $b_{i}$ by multiplying (A.16) by $\left(X-a_{i}\right)$ and by evaluating $X=a_{i}$ :

$$
b_{i}=-\prod_{\substack{j=1 \\ j \neq i}}^{s-1} \frac{1}{a_{i}-a_{j}}+\prod_{\substack{j=1 \\ j \neq i}}^{s-1} \frac{1}{a_{i}-a_{j}}=0
$$


We deduce that $F=0$. By remarking that

$$
F\left(a_{s}\right)=\sum_{i=1}^{s} \prod_{\substack{j=1 \\ j \neq i}}^{s} \frac{1}{a_{i}-a_{j}}=0
$$

we conclude the proof of (A.15)

The following result is an easy consequence of Lemma A.7.

Corollary A.8. For every $m+2 \leq k \leq n$, we have

$$
\sum_{l=m+1}^{k} P_{k l}\left(\beta_{l}-\alpha_{l}\right)=0
$$

Proof. By (2.2), we have by taking $s=k-m$ and $a_{i}=d_{i+m}$ for $1 \leq i \leq k-m$ in Lemma A.7

$$
\sum_{l=m+1}^{k} P_{k l}\left(\beta_{l}-\alpha_{l}\right)=\sum_{i=1}^{k-m} P_{k, i+m}\left(\beta_{i+m}-\alpha_{i+m}\right)=\sum_{i=1}^{k-m} \prod_{\substack{j=1 \\ j \neq i}}^{i-m} \frac{1}{d_{i+m}-d_{j+m}}=0 .
$$

This ends the proof of Corollary A.8.

Now, we turn to the proof of Proposition 2.1.

Proof. We introduce the following notation: $R:=\prod_{k=1}^{n} u_{k}^{\alpha_{k}}-\prod_{k=1}^{n} u_{k}^{\beta_{k}}$.

We assume that $\left(U, H^{J}\right)$ is a trajectory of (NL-U). The equations $1 \leq i \leq m+1$ of (NL-Z) are clearly satisfied. Let $m+2 \leq i \leq n$. We have:

$$
\begin{aligned}
\partial_{t} z_{i}-d_{i} \Delta z_{i} & =\partial_{t}\left(\sum_{j=m+1}^{i} P_{i j}\left(u_{j}-u_{j}^{*}\right)\right)-d_{i} \Delta\left(\sum_{j=m+1}^{i} P_{i j}\left(u_{j}-u_{j}^{*}\right)\right) \\
& =\sum_{j=m+1}^{i} P_{i j}\left(\partial_{t} u_{j}-d_{j} \Delta u_{j}+\left(d_{j}-d_{i}\right) \Delta u_{j}\right) \\
& =\sum_{j=m+1}^{i}(P_{i j}\left(\left(\beta_{j}-\alpha_{j}\right) R\right)+P_{i j} \underbrace{\left(d_{j}-d_{i}\right)}_{0 \text { if } j=i} \Delta u_{j}) \\
& =R \underbrace{\sum_{j=m+1}^{i} P_{i j}\left(\beta_{j}-\alpha_{j}\right)}_{0 \text { by Corollary A.8 }}+\sum_{j=m+1}^{i-1} \underbrace{P_{i j}\left(d_{j}-d_{i}\right)}_{P_{i-1, j} \text { by }(2.2)} \Delta u_{j} \\
& =\Delta z_{i-1} .
\end{aligned}
$$

This ends the proof of " $\Rightarrow$ ".

We assume that $\left(Z, H^{J}\right)$ satisfies (NL-Z). Then, the equations

$$
\partial_{t} u_{i}-d_{i} \Delta u_{i}=\left(\beta_{i}-\alpha_{i}\right) R,
$$


are clearly satisfied for $1 \leq i \leq m+1$. We prove (A.19) by strong induction on $i \in\{m+2, \ldots, n\}$. By using (A.18) for $i=m+2$ and (A.19) for $i=m+1$, we obtain

$$
\begin{aligned}
& \sum_{j=m+1}^{m+2} P_{m+2, j}\left(\partial_{t} u_{j}-d_{j} \Delta u_{j}\right)=0 \\
& \Leftrightarrow P_{m+2, m+2}\left(\partial_{t} u_{m+2}-d_{m+2} \Delta u_{m+2}\right)=-R P_{m+2, m+1}\left(\beta_{m+1}-\alpha_{m+1}\right) .
\end{aligned}
$$

This leads to (A.19) for $i=m+2$ by using $P_{m+2, m+1} / P_{m+2, m+2}=-\left(\beta_{m+2}-\alpha_{m+2}\right) /\left(\beta_{m+1}-\alpha_{m+1}\right)$ by $(2.2)$. For $i>m+2$, by induction, we have $P_{i i}\left(\partial_{t} u_{i}-d_{i} \Delta u_{i}\right)+\sum_{j=m+1}^{i-1} P_{i j}\left(\beta_{j}-\alpha_{j}\right) R=0$ by (A.18). Then, from Corollary A.8, we have $\sum_{j=m+1}^{i-1} P_{i j}\left(\beta_{j}-\alpha_{j}\right)=-P_{i i}\left(\beta_{i}-\alpha_{i}\right)$. This yields (A.19) and ends the proof of " $\Leftarrow$ ".

This concludes the proof of Proposition 2.1.

\section{A.4.2 Proof of the equivalence concerning the mass condition}

In this section, we prove the equivalence (2.7) which leads to the equivalence between Theorems 2.3 and 1.7.

Proof. Assume that $Z_{0} \in L_{\text {inv }}^{\infty}$. Then, we have

$$
\forall m+2 \leq i \leq n, \int_{\Omega} \sum_{k=m+1}^{i} P_{i k}\left(u_{k, 0}(x)-u_{k}^{*}\right) \mathrm{d} x=0
$$

We prove (1.18) by strong induction on $k \geq m+2$. The case $k=m+2$ comes from (A.20) for $i=m+2$ and $P_{m+2, m+1} / P_{m+2, m+2}=-\left(\beta_{m+2}-\alpha_{m+2}\right) /\left(\beta_{m+1}-\alpha_{m+1}\right)$ by (2.2). For $i>m+2$ in (A.20), by induction, we have

$$
\int_{\Omega}\left\{P_{i i}\left(u_{i, 0}(x)-u_{i}^{*}\right)+\sum_{k=m+1}^{i-1} P_{i k} \frac{\left(\beta_{k}-\alpha_{k}\right)\left(u_{m+1,0}(x)-u_{m+1}^{*}\right)}{\beta_{m+1}-\alpha_{m+1}}\right\} \mathrm{d} x=0 .
$$

Then, from Corollary A.8, we have $\sum_{k=m+1}^{i-1} P_{i k}\left(\beta_{k}-\alpha_{k}\right)=-P_{i i}\left(\beta_{i}-\alpha_{i}\right)$. This yields (1.18) for $k=i$.

Assume (1.18) holds. From Corollary A.8, we have that for every $m+2 \leq i \leq n$,

$$
\int_{\Omega} \sum_{k=m+1}^{i} P_{i k}\left(u_{k, 0}(.)-u_{k}^{*}\right)=\int_{\Omega} \sum_{k=m+1}^{i} P_{i k} \frac{\beta_{k}-\alpha_{k}}{\beta_{m+1}-\alpha_{m+1}}\left(u_{m+1,0}(.)-u_{m+1}^{*}\right)=0 .
$$

This ends the proof of $(2.7)$.

\section{A.5 Proof of an observability estimate for linear finite dimensional systems}

The goal of this section is to give a self-contained proof of Lemma 3.5. By the Hilbert Uniqueness Method (see [8], Thm. 2.44), it suffices to show the following null-controllability result for finite dimensional systems. 
Proposition A.9. There exist $C>0, p_{1}, p_{2} \in \mathbb{N}$ such that for every $\tau \in(0,1), \lambda \geq \lambda_{1}$ with $\lambda_{1}$ the first positive eigenvalue of $\left(-\Delta, H_{N e}^{2}(\Omega)\right), y_{0} \in \mathbb{R}^{n}$, there exists a control $h \in L^{2}\left(0, \tau ; \mathbb{R}^{m}\right)$ verifying

$$
\|h\|_{L^{2}\left(0, T ; \mathbb{R}^{m}\right)}^{2} \leq C\left(1+\frac{1}{\tau^{p_{1}}}+\lambda^{p_{2}}\right)\left\|y_{0}\right\|_{\mathbb{R}^{n}}^{2}
$$

such that the solution $y \in L^{2}\left(0, \tau ; \mathbb{R}^{n}\right)$ of

$$
\begin{cases}y^{\prime}=A y+B h, & \text { in }(0, \tau) \\ y(0)=y_{0} & \text { in } \mathbb{R}^{n}\end{cases}
$$

where $A=-\lambda D_{J}+A_{J}$ (see (2.5), (2.8) and (2.9)) and $B=\left(\begin{array}{c}I_{m} \\ (0)\end{array}\right) \in \mathcal{M}_{n, m}(\mathbb{R})$, satisfies $y(\tau)=0$.

Remark A.10. We do not treat the case $\lambda_{0}=0$ with initial data $y_{0} \in \mathbb{R}^{m+1} \times\{0\}^{n-m-1}$ because it is a simple adaptation of the following proof.

Proof. Let $\tau \in(0,1), \lambda \geq \lambda_{1}, y_{0} \in \mathbb{R}^{n}$.

Step 1: Construction of the control $h$ by a Brunovsky approach. We start by defining $\bar{y}$ to be the free solution of the system (A.22) (take $h=0)$. We have $\bar{y}(t)=e^{t A} y_{0}=e^{t\left(-\lambda D_{J}+A_{J}\right)} y_{0}$. We easily have that for any $l \geq 0$

$$
\left\|\bar{y}^{(l)}\right\|_{L^{2}\left(0, \tau ; \mathbb{R}^{n}\right)} \leq C\left(1+\lambda^{l-1 / 2}\right)\left\|y_{0}\right\|_{\mathbb{R}^{n}} .
$$

We choose a cut-off function $\eta \in C^{\infty}([0, \tau] ; \mathbb{R})$ such that $\eta=1$ on $[0, \tau / 3]$ and $\eta=0$ on $[2 \tau / 3, \tau]$ verifying:

$$
\forall p \in \mathbb{N}, \forall t \in[0, \tau],\left|\eta^{(p)}(t)\right| \leq \frac{C_{p}}{\tau^{p}} .
$$

We start by choosing for every $i \in\{1, \ldots, m-1, n\}$,

$$
y_{i}(t):=\eta(t) \overline{y_{i}}(t)
$$

Then, by using the cascade form of (A.22), we define by reverse induction on $i \in\{n-1, n-2, \ldots, m+1\}$,

$$
y_{i}(t):=-\frac{1}{\lambda}\left(y_{i+1}^{\prime}(t)+\lambda d_{i+1} y_{i+1}(t)\right)
$$

Then, $y_{m}$ is defined by the equation number $(m+1)$ by

$$
y_{m}(t):=\frac{1}{a_{m+1, m}}\left(y_{m+1}^{\prime}(t)+\lambda d_{m+1} y_{m+1}(t)-\sum_{\substack{s=1 \\ s \neq m}}^{n} a_{m+1, s} y_{s}(t)\right) .
$$

Finally, we set for the control

$$
h:=y^{\prime}-A y .
$$


By (A.27) and (A.28), $h$ is of the form $h=\left(h_{1}, \ldots, h_{m}, 0, \ldots, 0\right)$.

Step 2: Properties of the solution $y$ and estimate of the control $h$. First, we remark that,

$$
\forall 1 \leq i \leq n, \begin{cases}y_{i}=\overline{y_{i}}, & \text { in }[0, \tau / 3] \\ y_{i}=0, & \text { in }[2 \tau / 3, \tau]\end{cases}
$$

Indeed, the property (A.29) is clear for $i \in\{1, \ldots, m-1, n\}$ by definition (A.25). Then, we prove (A.29) by reverse induction on $m \leq i \leq n$ by using (A.26), (A.27) and the definition of $\bar{y}$, for instance, for $t \in[0, \tau / 3]$ :

$$
y_{n-1}(t)=-\frac{1}{\lambda}\left(y_{n}^{\prime}(t)+\lambda d_{n} y_{n}(t)\right)=-\frac{1}{\lambda}\left({\overline{y_{n}}}^{\prime}(t)+\lambda d_{n} \overline{y_{n}}(t)\right)=\overline{y_{n-1}}(t) .
$$

Now, we have by (A.25), (A.24) and (A.23) that for every $i \in\{1, \ldots, m-1\}$,

$$
\sum_{l=0}^{1}\left\|y_{i}^{(l)}\right\|_{L^{2}\left(0, \tau ; \mathbb{R}^{n}\right)} \leq C\left(1+\frac{1}{\tau^{1 / 2}}+\lambda^{1 / 2}\right)\left\|y_{0}\right\|_{\mathbb{R}^{n}}
$$

Then, we easily prove by reverse induction on $m \leq i \leq n$ by using (A.23), (A.24), (A.25), (A.26), (A.27) and (A.30)

$$
\sum_{l=0}^{i+1-m}\left\|y_{i}^{(l)}\right\|_{L^{2}\left(0, \tau ; \mathbb{R}^{n}\right)} \leq C\left(1+\frac{1}{\tau^{n-m+1 / 2}}+\lambda^{n-m+1 / 2}\right)\left\|y_{0}\right\|_{L^{2}\left(0, \tau ; \mathbb{R}^{n}\right)}
$$

Hence, the control $h$ and the state $y$ satisfy (A.21), (A.22) with $p_{1}=p_{2}=2(n-m+1 / 2)$ and $y(\tau)=0$.

\section{A.6 Source term method in $L^{r}$ for $r \in\{2,+\infty\}$}

We use the same notations as in the beginning of Section 4 . The goal of this section is to prove Propositions 4.3 and 5.3. We have the following result.

Proposition A.11. For every $S \in \mathcal{S}_{r}$ and $Z_{0} \in L_{\text {inv }}^{r}$, there exists $H^{J} \in \mathcal{H}_{r}$, such that the solution $Z$ of $(\mathrm{L}+\mathrm{S}-\mathrm{Z})$ satisfies $Z \in \mathcal{Z}_{r}$. Furthermore, there exists $C>0$, not depending on $S$ and $Z_{0}$, such that

$$
\left\|Z / \rho_{0}\right\|_{L^{\infty}\left([0, T] ; L^{r}(\Omega)^{n}\right)}+\left\|H^{J}\right\|_{\mathcal{H}_{r}} \leq C_{T}\left(\left\|Z_{0}\right\|_{L^{r}(\Omega)^{n}}+\|S\|_{\mathcal{S}_{r}}\right)
$$

where $C_{T}=C e^{C / T}$.

The proof is inspired by ([5], Prop. 2.6) and ([28], Prop. 2.3).

Proof. For $k \geq 0$, we define $T_{k}:=T\left(1-q^{-k}\right)$ where $q \in(1, \sqrt{2})$. On the one hand, let $a_{0}:=Z_{0}$ and, for $k \geq 0$, we define $a_{k+1}:=Z_{S}\left(T_{k+1}^{-},.\right)$where $Z_{S}$ is the solution to

$$
\begin{cases}\partial_{t} Z_{S}-D_{J} \Delta Z_{S}=A_{J} Z_{S}+S & \text { in }\left(T_{k}, T_{k+1}\right) \times \Omega, \\ \frac{\partial Z_{S}}{\partial \nu}=0 & \text { on }\left(T_{k}, T_{k+1}\right) \times \partial \Omega \\ Z_{S}\left(T_{k}^{+}, .\right)=0 & \text { in } \Omega .\end{cases}
$$


From Propositions A.2 and A.3, using the estimates (A.2) and (1.11) for $r=2$ or (A.3) and (1.11) for $r=+\infty$, we have

$$
\left\|a_{k+1}\right\|_{L^{r}(\Omega)^{n}} \leq\left\|Z_{S}\right\|_{L^{\infty}\left(\left[T_{k}, T_{k+1}\right] ; L^{r}(\Omega)^{n}\right)} \leq C\|S\|_{L^{r}\left(\left(T_{k}, T_{k+1}\right) ; L^{r}(\Omega)^{n}\right)} .
$$

On the other hand, for $k \geq 0$, we also consider the control systems

$$
\begin{cases}\partial_{t} Z_{H}-D_{J} \Delta Z_{H}=A_{J} Z_{H}+H^{J} 1_{\omega} & \text { in }\left(T_{k}, T_{k+1}\right) \times \Omega, \\ \frac{\partial Z_{H}}{\partial \nu}=0 & \text { on }\left(T_{k}, T_{k+1}\right) \times \partial \Omega, \\ Z_{H}\left(T_{k}^{+}, .\right)=a_{k} & \text { in } \Omega .\end{cases}
$$

Using Theorem 3.1 for $r=2$ or Theorem 5.1 for $r=+\infty$, we can define $H_{k}^{J} \in L^{r}\left(\left(T_{k}, T_{k+1}\right) \times \Omega\right)^{m}$ such that $Z_{H}\left(T_{k+1}^{-},.\right)=0$ and, thanks to the cost estimate (3.1) for $r=2$ or (5.1) for $r=+\infty$ (recalling that $\left.C_{T} \leq M e^{M / T}\right)$

$$
\left\|H_{k}^{J}\right\|_{L^{r}\left(\left(T_{k}, T_{k+1}\right) \times \Omega\right)^{m}} \leq M e^{\frac{M}{T_{k+1}-T_{k}}}\left\|a_{k}\right\|_{L^{2}(\Omega)^{n}} .
$$

In particular, for $k=0$, we have

$$
\left\|H_{0}^{J}\right\|_{L^{r}\left(\left(T_{0}, T_{1}\right) \times \Omega\right)^{m}} \leq M e^{\frac{q M}{T(q-1)}}\left\|Z_{0}\right\|_{L^{2}(\Omega)^{n}} .
$$

And, since $\rho_{0}$ is decreasing

$$
\left\|H_{0}^{J} / \rho_{0}\right\|_{L^{r}\left(\left(T_{0}, T_{1}\right) \times \Omega\right)^{m}} \leq \rho_{0}^{-1}\left(T_{1}\right) M e^{\frac{q M}{T(q-1)}}\left\|Z_{0}\right\|_{L^{2}(\Omega)^{n}} .
$$

For $k \geq 0$, since $\rho_{\mathcal{S}}$ is decreasing, combining (A.32) and (A.33) yields

$$
\left\|H_{k+1}^{J}\right\|_{L^{r}\left(\left(T_{k+1}, T_{k+2}\right) \times \Omega\right)^{m}} \leq C M e^{\frac{M}{T_{k+2}-T_{k+1}}} \rho_{\mathcal{S}}\left(T_{k}\right)\left\|S / \rho_{\mathcal{S}}\right\|_{L^{r}\left(\left(T_{k}, T_{k+1}\right) \times \Omega\right)^{n}} .
$$

In particular, by using $M e^{\frac{M}{T_{k+2}-T_{k+1}}} \rho_{\mathcal{S}}\left(T_{k}\right)=\rho_{0}\left(T_{k+2}\right)$ (see (4.1) and (4.2)), we have

$$
\left\|H_{k+1}^{J}\right\|_{L^{r}\left(\left(T_{k+1}, T_{k+2}\right) \times \Omega\right)^{m}} \leq C \rho_{0}\left(T_{k+2}\right)\left\|S / \rho_{\mathcal{S}}\right\|_{L^{r}\left(\left(T_{k}, T_{k+1}\right) \times \Omega\right)^{n}} .
$$

Then, from (A.36), by using the fact that $\rho_{0}$ is decreasing,

$$
\left\|H_{k+1}^{J} / \rho_{0}\right\|_{L^{r}\left(\left(T_{k+1}, T_{k+2}\right) \times \Omega\right)^{m}} \leq C\left\|S / \rho_{\mathcal{S}}\right\|_{L^{r}\left(\left(T_{k}, T_{k+1}\right) \times \Omega\right)^{n}} .
$$

As in the original proof, we can paste the controls $H_{k}^{J}$ for $k \geq 0$ together by defining

$$
H^{J}:=\sum_{k \geq 0} H_{k}^{J} 1_{\left(T_{k}, T_{k+1}\right)}
$$

We have the estimate from (A.34) and (A.37)

$$
\left\|H^{J}\right\|_{\mathcal{H}_{r}} \leq C\|S\|_{\mathcal{S}_{r}}+C \rho_{0}^{-1}\left(T_{1}\right) M e^{\frac{q M}{T(q-1)}}\left\|Z_{0}\right\|_{L^{2}(\Omega)^{n}} .
$$


The state $Z$ can also be reconstructed by concatenation of $Z_{S}+Z_{H}$, which are continuous at each junction $T_{k}$ thanks to the construction. Then, we estimate the state. We use the energy estimate (A.2) for $r=2$ or (A.3) for $r=+\infty$ on each time interval $\left(T_{k}, T_{k+1}\right)$ :

$$
\left\|Z_{S}\right\|_{L^{\infty}\left(T_{k}, T_{k+1} ; L^{r}(\Omega)^{n}\right)} \leq C\|S\|_{L^{r}\left(\left(T_{k}, T_{k+1}\right) \times \Omega\right)^{n}},
$$

and

$$
\left\|Z_{H}\right\|_{L^{\infty}\left(T_{k}, T_{k+1} ; L^{r}(\Omega)^{n}\right)} \leq C\left(\left\|a_{k}\right\|_{L^{r}(\Omega)^{n}}+\left\|H_{k}^{J}\right\|_{L^{r}\left(\left(T_{k}, T_{k+1}\right) \times \Omega\right)^{m}}\right) .
$$

Proceeding similarly as for the estimate on the control, we obtain respectively

$$
\left\|Z_{S} / \rho_{0}\right\|_{L^{\infty}\left(T_{k}, T_{k+1} ; L^{r}(\Omega)^{n}\right)} \leq C M^{-1}\|S\|_{\mathcal{S}_{r}},
$$

and

$$
\left\|Z_{H} / \rho_{0}\right\|_{L^{\infty}\left(T_{k}, T_{k+1} ; L^{r}(\Omega)^{n}\right)} \leq C M^{-1}\|S\|_{\mathcal{S}_{r}}+C \rho_{0}^{-1}\left(T_{1}\right) M e^{\frac{q M}{T(q-1)}}\left\|Z_{0}\right\|_{L^{\infty}(\Omega)^{n}} .
$$

Therefore, for an appropriate choice of constant $C>0, Z$ and $H^{J}$ satisfy (A.31). This concludes the proof of Proposition A.11.

\section{A.7 Proof of a strong observability inequality}

We take the same notations as in the beginning of Section 4. The goal of this section is to prove Corollary 4.4.

Proof. We define $\mathcal{F}_{1}:\left(Z_{0}, S\right) \in L_{\text {inv }}^{2} \times \mathcal{S}_{2} \mapsto Z(T,.) \in L_{\text {inv }}^{2}$, where $Z$ is the solution of (L+S-Z) with $H^{J}=0$ and $\mathcal{F}_{2}: H^{J} \in \mathcal{H}_{2} \mapsto Z(T,.) \in L_{\text {inv }}^{2}$ is the solution of (L+S-Z) with $\left(Z_{0}, S\right)=(0,0)$. It is easy to see that the null-controllability of $(\mathrm{L}+\mathrm{S}-\mathrm{Z})$ is equivalent to $\operatorname{Range}\left(\mathcal{F}_{1}\right) \subset \operatorname{Range}\left(\mathcal{F}_{2}\right)$.

From ([8], Lem. 2.48), we have that $\operatorname{Range}\left(\mathcal{F}_{1}\right) \subset \operatorname{Range}\left(\mathcal{F}_{2}\right)$ is equivalent to the observability inequality

$$
\exists C_{T}>0, \forall \varphi_{T} \in L_{\mathrm{inv}}^{2},\left\|\mathcal{F}_{1}^{*}\left(\varphi_{T}\right)\right\|_{L_{\mathrm{inv}}^{2} \times \mathcal{S}_{2}} \leq C_{T}\left\|\mathcal{F}_{2}^{*}\left(\varphi_{T}\right)\right\|_{\mathcal{H}_{2}} .
$$

Consequently, by using the null-controllability result for (L+S-Z): Proposition 4.3, we have that (A.38) holds true. Moreover, the constant $C_{T}$ in (A.38) can be chosen such that $C_{T} \leq C e^{C / T}$ by using the cost estimate (4.7) (see the proof of [8], Thm. 2.44 for more details between the constant of cost estimate and the constant of observability inequality).

Duality $\operatorname{arguments}$ between $Z$, the solution of $(\mathrm{L}+\mathrm{S}-\mathrm{Z})$, and $\varphi$, the solution of (4.8), lead to:

$$
\begin{gathered}
\int_{\Omega} \mathcal{F}_{1}\left(Z_{0}, S\right)(x) \cdot \varphi_{T}(x) \mathrm{d} x=\int_{\Omega} Z_{0}(x) \cdot \varphi(0, x) \mathrm{d} x+\iint_{(0, T) \times \Omega} S \cdot \varphi \\
\left(\left(Z_{0}, S\right), \mathcal{F}_{1}^{*}\left(\varphi_{T}\right)\right)_{L^{2}(\Omega)^{n} \times \mathcal{S}_{2}}=\int_{\Omega} Z_{0}(x) \cdot \varphi(0, x) \mathrm{d} x+\iint_{(0, T) \times \Omega} S \cdot \varphi \rho_{\mathcal{S}}^{2} \rho_{\mathcal{S}}^{-2} \\
\int_{\Omega} \mathcal{F}_{2}\left(H^{J}\right)(x) \cdot \varphi_{T}(x) \mathrm{d} x=\iint_{(0, T) \times \omega} H^{J} \cdot \varphi \\
\left(H^{J}, \mathcal{F}_{2}^{*}\left(\varphi_{T}\right)\right)_{\mathcal{H}_{2}}=\sum_{i=1}^{m} \iint_{(0, T) \times \Omega} h_{i} \cdot \varphi_{i} \rho_{0}^{2} 1_{\omega} \rho_{0}^{-2} .
\end{gathered}
$$


Consequently, by identification, we find

$$
\mathcal{F}_{1}^{*}\left(\varphi_{T}\right)=\left(\varphi(0, .), \varphi \rho_{\mathcal{S}}^{2}\right) \in L^{2}(\Omega)^{n} \times \mathcal{S}_{2}, \quad \mathcal{F}_{2}^{*}\left(\varphi_{T}\right)=\left(\varphi_{i} \rho_{0}^{2} 1_{\omega}\right)_{1 \leq i \leq m} \in \mathcal{H}_{2} .
$$

Finally, by putting (A.39) in (A.38), we exactly obtain (4.9) with $C_{T}=C e^{C / T}$. This ends the proof of Corollary 4.4.

Acknowledgements. I would like to very much thank Karine Beauchard and Michel Pierre (Ecole Normale Supérieure de Rennes) for many fruitful, stimulating discussions, helpful advices. I am grateful to Joackim Bernier (Université de Rennes 1) for suggesting me the proof of Lemma A.7. I also thank the two anonymous referees that provided a lot of comments, remarks that improved the article.

\section{REFERENCES}

[1] F. Ammar-Khodja, A. Benabdallah, C. Dupaix and M. González-Burgos, A Kalman rank condition for the localized distributed controllability of a class of linear parbolic systems. J. Evol. Equ. 9 (2009) 267-291.

[2] F. Ammar-Khodja, A. Benabdallah, M. González-Burgos and L. de Teresa, Recent results on the controllability of linear coupled parabolic problems: a survey. Math. Control Relat. Fields 1 (2011) 267-306.

[3] V. Barbu, Local controllability of the phase field system. Nonlinear Anal. 50 (2002) 363-372.

[4] C. Bardos and L. Tartar, Sur l'unicité rétrograde des équations paraboliques et quelques questions voisines. Arch. Ration. Mech. Anal. 50 (1973) 10-25.

[5] K. Beauchard and F. Marbach, Unexpected quadratic behaviors for the small-time local null controllability of scalar-input parabolic equations. Preprint arXiv:1712.09790 (2017).

[6] C. Caputo, T. Goudon and A.F. Vasseur, Solutions of the 4-species quadratic reaction-diffusion system are bounded and $C^{\infty}$, in any space dimension. Preprint arXiv:1709.05694 (2017).

[7] J.-M. Coron, Global asymptotic stabilization for controllable systems without drift. Math. Control Signals Systems 5 (1992) 295-312.

[8] J.-M. Coron, Control and nonlinearity, Mathematical Surveys and Monographs. American Mathematical Society, Providence, RI (2007), p. 136.

[9] J.-M. Coron, S. Guerrero, P. Martin and L. Rosier, Homogeneity applied to the controllability of a system of parabolic equations, in Proceedings 2015 European Control Conference (ECC 2015), Linz, Austria (2015) 2470-2475.

[10] J.-M. Coron, S. Guerrero and L. Rosier, Null controllability of a parabolic system with a cubic coupling term. SIAM J. Control Optim. 48 (2010) 5629-5653.

[11] R. Denk, M. Hieber and J. Prüss, Optimal $L^{p}-L^{q}$-estimates for parabolic boundary value problems with inhomogeneous data. Math. Z. 257 (2007) 193-224.

[12] E. Fernández-Cara, A review of basic theoretical results concerning the Navier-Stokes and other similar equations. Bol. Soc. Esp. Mat. Apl. SeMA 32 (2005) 45-73.

[13] E. Fernández-Cara and S, Guerrero, Global Carleman inequalities for parabolic systems and applications to controllability. SIAM J. Control Optim. 45 (2006) 1399-1446.

[14] E. Fernández-Cara, J. Limaco and S.B. de Menezes, Controlling linear and semilinear systems formed by one elliptic and two parabolic PDEs with one scalar control. ESAIM: COCV 22 (2016) 1017-1039.

[15] J. Fischer, Global existence of renormalized solutions to entropy-dissipating reaction-diffusion systems. Arch. Ration. Mech. Anal. 218 (2015) 553-587.

[16] A.V. Fursikov and O. Yu. Imanuvilov. Controllability of evolution equations, Vol. 34 of Lecture Notes Series. Seoul National University, Research Institute of Mathematics, Global Analysis Research Center, Seoul (1996).

[17] P. Gao, Null controllability with constraints on the state for the reaction-diffusion system. Comput. Math. Appl. 70 (2015) $776-788$.

[18] M. González-Burgos and L. de Teresa, Controllability results for cascade systems of $m$ coupled parabolic PDEs by one control force. Port. Math. 67 (2010) 91-113.

[19] O. Imanuvilov and T. Takahashi, Exact controllability of a fluid-rigid body system. J. Math. Pures Appl. 87 (2007) $408-437$.

[20] D. Jerison and G. Lebeau. Nodal sets of sums of eigenfunctions, in Harmonic analysis and partial differential equations (Chicago, IL, 1996), Chicago Lectures in Mathematics, University of Chicago Press, Chicago, IL (1999) $223-239$.

[21] J. Kanel, The Cauchy problem for a system of semilinear parabolic equations with balance conditions. Differentsial nye Uravneniya 20 (1984) 1753-1760.

[22] J. Kanel, Solvability in the large of a system of reaction-diffusion equations with the balance condition. Differentsial nye Uravneniya, 26 (1990) 448-458.

[23] K. Le Balc'h, Null-controllability of two species reaction-diffusion system with nonlinear coupling: a new duality method. Preprint arXiv:1802.09187 (2018).

[24] K. Le Balc'h, Controllability of a $4 \times 4$ quadratic reaction-diffusion system. J. Differ. Equ. 266 (2019) 3100-3188. 
[25] J. Le Rousseau and G. Lebeau, On Carleman estimates for elliptic and parabolic operators. Applications to unique continuation and control of parabolic equations. ESAIM: COCV 18 (2012) 712-747.

[26] G. Lebeau and L. Robbiano, Contrôle exact de l'équation de la chaleur. In Séminaire sur les Équations aux Dérivées Partielles, 1994-1995, pages Exp. No. VII, 13. École Polytech., Palaiseau (1995).

[27] P. Lissy and E. Zuazua, Internal observability for coupled systems of linear partial differential equations. Preprint hal-01480301 (2018).

[28] Y. Liu, T. Takahashi and M. Tucsnak, Single input controllability of a simplified fluid-structure interaction model. ESAIM: COCV 19 (2013) 20-42.

[29] S. Micu and T. Takahashi, Local controllability to stationary trajectories of a Burgers equation with nonlocal viscosity. $J$. Differ. Equ. 264 (2018) 3664-3703.

[30] B. Perthame, Parabolic equations in biology. Growth, reaction, movement and diffusion. Lecture Notes on Mathematical Modelling in the Life Sciences. Springer, Cham, 2015.

[31] M. Pierre, Global existence in reaction-diffusion systems with control of mass: a survey. Milan J. Math. 78 (2010) 417-45.

[32] T.I. Seidman, How violent are fast controls? Math. Control Signals Syst. 1 (1988) 89-95.

[33] P. Souplet, Global existence for reaction-diffusion systems with dissipation of mass and quadratic growth. J. Evolut. Equ. 18 (2018) 1713-1720.

[34] Z. Wu, J. Yin and C. Wang, Elliptic \& parabolic equations. World Scientific Publishing Co. Pte. Ltd., Hackensack, NJ (2006). 